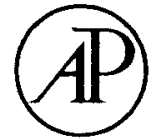

ACADEMIC

PRESS
Available online at www.sciencedirect.com

BCience@inect

Journal of Sound and Vibration 264 (2003) 657-687
JOURNAL OF

SOUND AND

VIBRATION

www.elsevier.com/locate/jsvi

\title{
Non-linear structural dynamics characterization using a scanning laser vibrometer
}

\author{
P.F. Pai*, S.-Y. Lee \\ Department of Mechanical and Aerospace Engineering, Unicersity of Missouri-Columbia, Columbia, MO 65211, USA
}

Received 28 September 2001: accepted 9 July 2002

\begin{abstract}
This paper presents the use of a scanning laser vibrometer and a signal decomposition method to characterize non-linear dynamics of highly flexible structures. A Polytec PI PSV-200 scanning laser vibrometer is used to measure transverse velocities of points on a structure subjected to a harmonic excitation. Velocity profiles at different times are constructed using the measured velocities, and then each velocity profile is decomposed using the first four linear mode shapes and a least-squares curve-fitting method. From the variations of the obtained modal velocities with time we search for possible non-linear phenomena. A cantilevered titanium alloy beam subjected to harmonic base-excitations around the second, third, and fourth natural frequencies are examined in detail. Influences of the fixture mass, gravity, mass centers of mode shapes, and non-linearities are evaluated. Geometrically exact equations governing the planar, harmonic large-amplitude vibrations of beams are solved for operational deflection shapes using the multiple shooting method. Experimental results show the existence of $1: 3$ and 1:2:3 external and internal resonances, energy transfer from high-frequency modes to the first mode, and amplitude- and phasemodulation among several modes. Moreover, the existence of non-linear normal modes is found to be questionable.
\end{abstract}

C 2002 Elsevier Science Ltd. All rights reserved.

\section{Introduction}

Because the cargo space of a launch vehicle is always limited, large space structures (e.g., solar collectors, dish antennas, radiators, sun shields, habitats, radio-frequency structures, optical communication systems, radars, lightweight radiometers, and telescopes) must be designed to be stowed during launch and deployed once on orbit. Moreover, because the launch expenditure of a NASA space mission always constitutes a significant fraction of the total cost, inexpensive launch

${ }^{*}$ Corresponding author. Tel.: + 1-573-884-1474; fax: + 1-573-884-5090.

E-mail address: paip a missouri.edu (P.F. Pai). 
vehicles with small payload masses are always desirable. Hence, instead of using previous electromechanical types of deploying systems, recent efforts of NASA concentrate on the use of highly flexible ultra-lightweight deployable/inflatable structures for space applications [1]. These recent developments have stimulated extensive research into the mechanics and dynamics of highly flexible structures.

Highly flexible deployable/inflatable structures are made of thin-walled structures and membranes. Because these structures usually have small material damping and there is no environmental damping in space, maneuver often leads to destructive large vibrations, which affect the operational accuracy, increase the operation expense, and reduce the life of structures. Because highly flexible structures can undergo large displacements and rotations without exceeding their elastic limits, geometrically exact modelling and accurate computational methods are needed in order to understand the behaviors of such structures, to evaluate their actual load carrying capacity, and to determine an efficient control method [1-3].

Non-linear models are required in analyzing actual flexible structures, such as helicopter rotor blades, wind turbine blades, and most space and aerospace structures. A linear static model of a structure can predict the onset of static bifurcation (buckling) but cannot predict post-buckling displacements. In a pseudo-non-linear model, the static behavior is described by a non-linear model, but the dynamic behavior is described by a linear model. The non-linear static model is used to predict the static equilibrium configuration after buckling, and the linear dynamic model describes vibrations around the static equilibrium position and is used to perform dynamic stability analysis and to predict the onset of dynamic bifurcation. However, a linear dynamic model cannot predict limit cycles or chaotic attractors, which occur after dynamic bifurcation. Hence, a geometrically exact (or fully non-linear) structural model that can describe large static and dynamic deformations is required for the study of highly flexible structures.

Software developed for the design and analysis of highly flexible structures needs to be experimentally verified for its applicability and accuracy. Geometrically exact modelling, analysis, and experimental verification of large static deformations of highly flexible structures have been demonstrated by many researchers [4-6]. However, obtaining accurate large dynamic deformations of highly flexible structures is still a very challenging task, and experimental verification of large dynamic deformations is even more difficult. To solve non-linear dynamic problems of actual structures without using the finite-element method is almost impossible. Although the finite-element method is still the most popular method for analyzing complex structures because of its systematic approach of treating different structural elements and system boundaries, finiteelement solutions are always approximate answers because of the use of polynomial shape functions and variational formulations. Moreover, using different stress and strain measures, different methods of meshing the geometric domain, different iteration methods in solving nonlinear algebra equations, and even different methods of tracing equilibrium paths can result in different solution errors in finite-element analyses. Hence, experimental verification of non-linear dynamic responses from finite element analyses is important in order to assure the performance of a non-linear finite-element code. Unfortunately, large-amplitude vibration testing itself is very challenging. Because of significant mass and stiffness, wiring, conductivity requirement, and/or limited measurement range, conventional sensors (such as accelerometers, strain gauges, and displacement sensors using eddy current) are not suitable for measuring large dynamic responses of highly flexible structures. 
In this work a scanning laser vibrometer is used to provide non-contact measurements of velocities of many points on a cantilevered beam, and a signal decomposition method is developed to process these velocities to reveal non-linear characteristics, such as superharmonic and subharmonic external resonances, internal resonances, amplitude- and phase-modulated motions, and energy transfer from high- to low-frequency modes. Moreover, large-amplitude operational deflection shapes are obtained by solving geometrically exact equations of motion using the multiple shooting method, and the concept of non-linear normal modes is examined.

\section{Experimental set-up}

Fig. 1 shows the set-up of a Polytec PSV-200 scanning laser vibrometer for measuring velocities of many equally spaced points on a beam, where the Ling Dynamics LDS V408 shaker has a maximum output force of $196 \mathrm{~N}$ and can provide $5-9000 \mathrm{~Hz}$ excitations. The PSV-200 scanning laser vibrometer can provide non-contact (measuring velocities of a dynamic system by checking the frequency shift of a back-scattered laser beam), remote (up to $30 \mathrm{~m}$ away), large-area scanning (up to $40^{\circ} \times 40^{\circ}$ ), dense (up to $512 \times 512$ points), high-frequency bandwidth $(0.2 \mathrm{~Hz}-20 \mathrm{MHz}$ ), and accurate (a velocity resolution of $0.1 \mu \mathrm{m} / \mathrm{s}$ ) measurements. The vibrometer system comprises an OFV-055 optical scan head, an OFV-3001-S controller, a video control box, and a Pentium-II $400 \mathrm{MHz}$ computer system. The LDS PA500L power amplifier magnifies the AC voltage from the

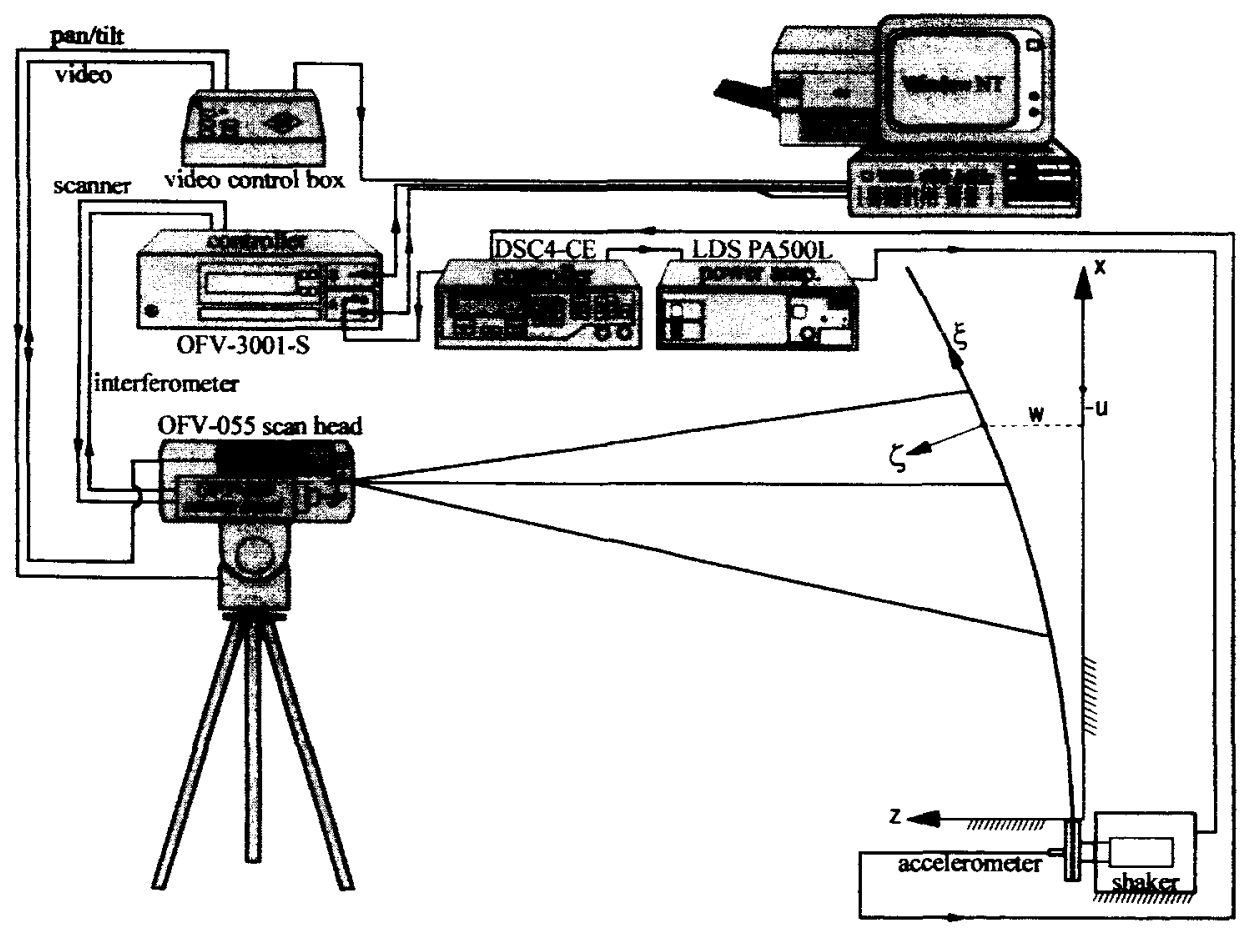

Fig. 1. The experimental set-up and the two co-ordinate systems used in the modelling. 
DSC4-CE shaker controller and sends it to the shaker to excite the structure. The PCB J353B03 shear ICP accelerometer feeds back the base acceleration to the DSC4-CE, and the DSC4-CE modifies the AC voltage sent to the LDS PA500L to keep the base motion harmonic. The scanning head launches a probe beam to the structure's surface and collects the back-scattered light signal as well. The OFV-3001-S vibrometer controller provides power for the scanning head, controls the rotation of the two mirrors in the OFV-055 scan head and the scanning of the laser beam, and processes the interferometry created by the back-scattered laser beam and the reference laser beam in the OFV-303 sensor head. The output voltage from the DSC4-CE is also taken as the input signal to the structure by Channel A of the OFV-3001-S vibrometer controller and is also used as the signal for triggering data acquisition by the computer. The OFV-3001-S controller includes two independently programmable low-pass filters for filtering the signals from the DSC4$\mathrm{CE}$ and the OFV-303 sensor head, respectively. After filtering, these two signals are sent from Channels A and B of the vibrometer controller to the $400 \mathrm{MHz}$ computer system, which is operated by Windows NT and processes the measured data. The PSV software in the computer system controls the entire measuring system, which includes the high-speed FFT processor, analog-to-digital converters, laser focus and position, vibrometer electronics, and a live video system. The video control box controls the swiveling and tilting of the OFV-055 scan head and the focusing of the video camera in the OFV-055 scan head. A standardized composite video signal from the camera is passed via a BNC connection on the video control box to the video input of the computer system. The exact area to be scanned can be drawn with reference to the video image of the structure on the monitor.

When using the laser vibrometer to detect the surface velocity of a structure, bright spots in the speckle pattern of the laser light back-scattered from the surface will randomly fall on the optical sensor. This inherent fluctuation in the light intensity at the optical sensor is called speckle noise, and it depends on the ratio of the laser wavelength to the structural surface roughness. The PSV200 laser vibrometer employs an analog phase lock loop (PLL) circuit to demodulate the FMsignal. It is often that the speckle noise causes the PLL to lose its locking state so as to yield noise signals with large amplitudes. Speckle noise amplitudes from 2 to 10 times the expected velocity amplitudes may occur and the input to the A/D converter will become overloaded. Speckle noise occurs more often when the surface velocity is of low amplitude, the laser beam is improperly focused, the surface is of poor reflective nature, or the laser beam is off the structure's normal direction by a large angle. It will strongly limit the system performance in poor signal-to-noise ratio, short stand-off distance, and high velocity signals. To reduce spectral noise and reflection when the laser beam was not perpendicular to the structure due to dynamic deflection, a thin layer of retro-reflective tape was adhered to the beam shown in Fig. 1.

In the experiments we first performed an "FFT" acquisition to obtain frequency response functions (FRFs) using a small periodic chirp base-excitation, and then we obtained linear natural frequencies from the averaged FRF. After that we performed single-frequency large-amplitude base-excitations and examined non-linear vibration characteristics of the beam.

Fig. 2 shows that the laser beam does not really shoot at the same point because of the axial displacement $u$ (due to the shortening effect) and the non-zero shooting angle $\theta$ of the laser beam. To reduce this effect we set the OFV-055 scan head $2.4 \mathrm{~m}$ away from the beam to make $\theta<6^{\circ}$. Moreover, because this effect is especially significant if it is a large-amplitude first-mode vibration, and because the beam's first natural frequency (about $1.5 \mathrm{~Hz}$ ) is much lower than the minimum 


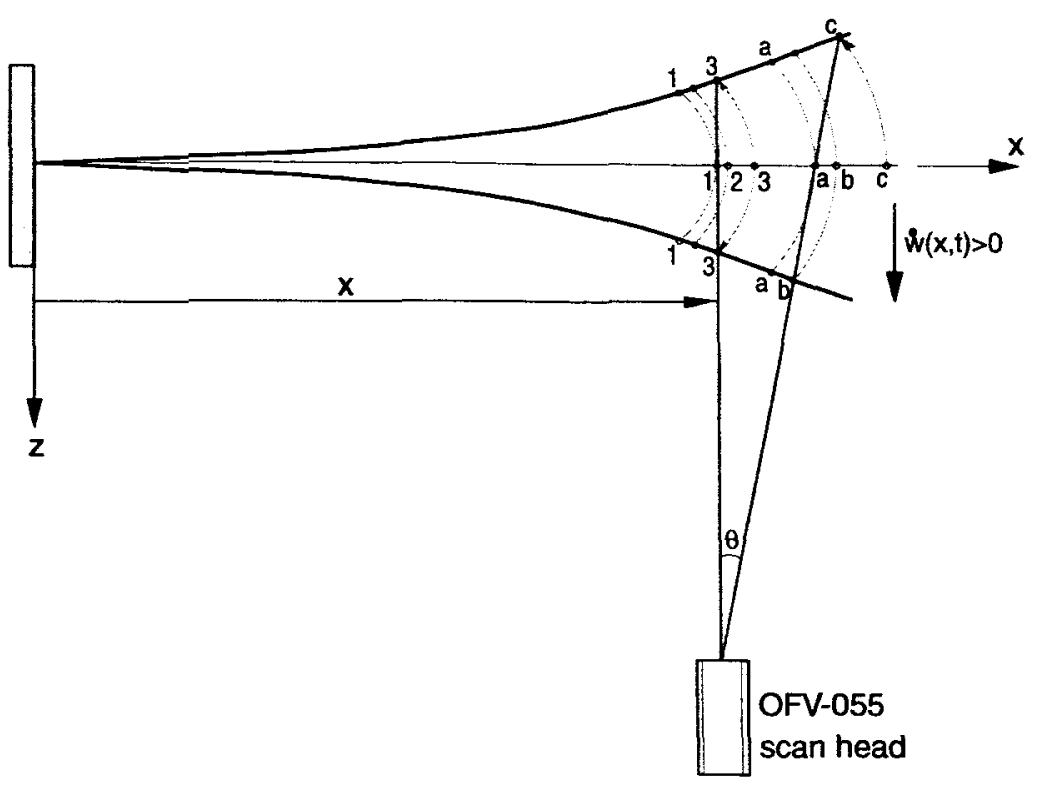

Fig. 2. The relation between the laser beam and measurement points.

frequency limit $(5 \mathrm{~Hz})$ of the shaker, large-amplitude first-mode vibrations are not examined in this study. For all the cases studied, the influence of $u$ is shown to be negligible by experimental results presented later in Section 5.

\section{Governing equations}

\subsection{Large-amplitude vibration}

To describe large deformations of a beam, two co-ordinate systems are needed; the system $x y z$ shown in Fig. 1 describes the undeformed system configuration and the system $\xi \xi \xi$ describes the deformed system configuration. The system $x y z$ is an inertial orthogonal rectilinear co-ordinate system in which the axis $x$ is the reference line formed by connecting the reference points of all cross-sections of the undeformed beam; and the system $\xi \eta \zeta$ is a local orthogonal curvilinear coordinate system in which the axis $\xi$ represents the deformed reference line and the axes $\eta$ and $\zeta$ represent the deformed configurations of the axes $y$ and $z$ in the absence of out-of-plane warpings. The unit vectors along the axes $x, z, \xi$, and $\zeta$ will be denoted by $\mathbf{i}_{x}, \mathbf{i}_{z}, \mathbf{i}_{1}$, and $\mathbf{i}_{3}$, respectively. $u$ and $w$ represent the displacement components of the reference point on the observed cross-section with respect to the axes $x$ and $z$, respectively.

The fully non-linear equations governing the two-dimensional motion of a highly flexible beam can be derived by using Jaumann stress and strain measures and an exact co-ordinate 
transformation to be [7]

$$
\begin{aligned}
F_{1}^{\prime} & =-\rho_{2} F_{3}-T_{11} q_{1}-T_{13} q_{3}+T_{11} m \ddot{u}+T_{13} m \ddot{u}, \\
F_{3}^{\prime} & =\rho_{2} F_{1}+T_{13} q_{1}-T_{11} q_{3}-T_{13} m \ddot{u}+T_{11} m \ddot{w}, \\
M_{2}^{\prime} & =(1+e) F_{3}, \quad T_{11}^{\prime}=\left(\rho_{2}-k_{2}\right) T_{13}, \quad T_{13}^{\prime}=-\left(\rho_{2}-k_{2}\right) T_{11}, \\
u^{\prime} & =-1-w k_{2}+(1+e) T_{11}, \quad w^{\prime}=u k_{2}+(1+e) T_{13},
\end{aligned}
$$

where ()$^{\prime} \equiv \partial() / \partial x,() \equiv \partial() / \partial t, F_{1}$ is the internal force along $\xi, F_{3}$ is the internal force along $\zeta, M_{2}$ is the internal bending moment along $\eta, \mathbf{i}_{1}=T_{11} \mathbf{i}_{x}+T_{13} \mathbf{i}_{z}\left(=\left(1+u^{\prime}+w k_{2}\right) /(1+e) \mathbf{i}_{x}+\right.$ $\left.\left(w^{\prime}-u k_{2}\right) /(1+e) \mathbf{i}_{z}\right), T_{11}^{2}+T_{13}^{2}=1, m$ is the mass per unit length, $e$ is the axial strain on and along the $\xi$ axis, $\rho_{2}$ is the normalized bending curvature along $\eta, q_{1}$ and $q_{3}$ are the distributed external loads along $x$ and $z$, respectively, and $k_{2}$ is the undeformed bending curvature along $y . k_{2}$ can be used to describe the geometry of an initially crooked beam. For an initially straight beam (such as the beam studied in this paper), $k_{2}=0$. Moreover, the constitutive equations are

$$
\rho_{2}=\frac{M_{2}}{E I}+k_{2}, \quad e=\frac{F_{1}}{E A}
$$

where $I$ is the area moment of inertia with respect to the $\eta$ axis, $E$ is Young's modulus, and $A$ is the cross-sectional area.

Because the base (i.e., the fixture) in Fig. 1 is confined to move horizontally, we have

$$
w(x, t)=b(t)+\bar{w}(x, t)
$$

where $b(t)$ denotes the base motion and $\bar{w}$ is the relative displacement of the beam with respect to the base. If $w(=b(t)+\bar{w}(x, t))$ is assumed to be harmonic when $b(t)$ is harmonic and $m \ddot{u}$ is assumed to be negligible, one can transform the non-linear dynamic problem in Eq. (1) into a quasi-static one by using

$$
\begin{aligned}
& k_{2}=0, \quad q_{3}=0, \quad q_{1}=-m g, \quad \ddot{w}=-\Omega^{2}(b+\bar{w}), \\
& w^{\prime}=\bar{w}^{\prime}, \quad w^{\prime \prime}=\bar{w}^{\prime \prime}, \quad w^{\prime \prime \prime}=\bar{w}^{\prime \prime \prime}, \quad w^{i v}=\bar{w}^{i v},
\end{aligned}
$$

where $g$ is the gravity and $\Omega$ is the excitation frequency. The problem is equivalent to a fixed-free vertical beam being loaded with a constant, distributed transverse load $m b \Omega^{2}$, a parametric load $m \overline{1} \Omega^{2}$, and the structural weight. The boundary conditions are

$$
T_{11}=1, T_{13}=u=\bar{w}=0 \text { at } x=0 ; F_{1}=F_{3}=M_{2}=0 \text { at } x=L,
$$

where $L$ is the beam length. Eqs. (1), (4), and (5) can be solved for $\bar{w}$ using the multiple shooting method [5,8]. The so-obtained $b+\bar{w}$ is called the operational deflection shape at the specific excitation frequency, and it can be used to examine the concept of non-linear normal modes. If the $\bar{w}$ is assumed to be the shape of a non-linear normal mode, the corresponding velocity profile is $\Omega(b+\bar{w})$.

\subsection{Weakly non-linear vibration}

If the vibration is weakly non-linear and the beam is assumed to be inextensible (i.e., $e=0$ ), one can perform Taylor's expansion to expand the longitudinal displacement $u$ and the bending 
curvature $\rho_{2}$ up to cubic non-linearity as [9]

$$
\begin{aligned}
& u=-\frac{1}{2} \int_{0}^{x} w^{\prime 2} \mathrm{~d} x, \\
& \rho_{2}=w^{\prime \prime}\left(1+\frac{1}{2} w^{\prime 2}\right) .
\end{aligned}
$$

And, the equation of motion is given by [9]

$$
m \ddot{w}=-E I\left(w^{\prime \prime \prime}+w^{\prime} w^{\prime \prime 2}+w^{\prime \prime \prime} w^{\prime 2}\right)^{\prime}+\left(w^{\prime} \int_{L}^{x} m \ddot{u} \mathrm{~d} s\right)^{\prime}+\left[w^{\prime \prime}(x-L)+w^{\prime}\right] m g .
$$

Substituting $\ddot{w}=-\Omega^{2}(b+\bar{w})$ and $w^{\prime}=\bar{w}^{\prime}$ from Eq. (4) into Eq. (8), neglecting $\ddot{u}$, and using the boundary conditions

$$
\bar{w}=\bar{w}^{\prime}=0 \quad \text { at } x=0, \quad \bar{w}^{\prime \prime}=\bar{w}^{\prime \prime \prime}=0 \quad \text { at } x=L,
$$

one can use the multiple shooting method to solve Eqs. (8) and (9) for operational deflection shapes. One can also solve Eqs. (8) and (9) for asymptotic solutions using perturbation methods [10]. However, a linear mode is included in a perturbation solution only if its natural frequency $\omega$ is commensurable with the excitation frequency $\Omega$ (i.e., external resonances), or $\omega$ is commensurable with the natural frequency of an excited mode (i.e., internal resonances). Because there are only cubic non-linearities in Eq. (8), first-order perturbation solutions of Eq. (8) can only predict 1:1 and 1:3 internal and external resonances. Moreover, the scaling parameter $\varepsilon$ used in a perturbation method for ordering is usually a mystery, and it is difficult to quantify the applicable parameter range of the perturbation solution obtained under a specific scaling. We will examine the applicability of Eq. (8) to large-amplitude analyses.

\section{Response to harmonic excitations}

We consider the upwardly cantilevered beam shown in Fig. 1, which is a $479.0 \times 50.8 \times$ $0.45 \mathrm{~mm}$ titanium alloy beam having a mass density $4430 \mathrm{~kg} / \mathrm{m}^{3}$, Young's modulus $127 \mathrm{GPa}$, and the Poisson ratio 0.36. Moreover, the effective moving mass (including the armature and the fixture base) of the shaker is given by the manufacturer to be $200 \mathrm{~g}$, and the fixture weighs $768 \mathrm{~g}$.

The free undamped linear vibration of a beam is governed by

$$
m \ddot{i}+E I w^{i n}=0 .
$$

Substituting Eq. (3) into Eq. (10) yields

$$
m \ddot{\bar{w}}+E I_{w}^{i v}=-m \ddot{b}(t) .
$$

If the excitation force on the movable part (including the effective moving mass of the shaker and the fixture) of the excitation system is assumed to be a harmonic force $F_{0} \sin \Omega t$, the equation of motion of the movable part is

$$
F_{0} \sin \Omega t-E w^{\prime \prime \prime}(0, t)=M \ddot{b}(t),
$$

where the mass $M(=0.2+0.768=0.968 \mathrm{Kg})$ is the mass of the movable part. Hence, it follows from Eqs. (11) and (12) that the equation governing the motion of the beam and the movable part 
is given by

$$
m \ddot{\bar{w}}+c \dot{\bar{w}}+E I w^{i r}=\frac{m}{M}\left[E I w^{\prime \prime \prime}(0, t)-F_{0} \sin \Omega t\right],
$$

where we added the damping term $c \dot{\bar{v}}$ and $c$ is the damping coefficient. Because Eq. (13) is linear and the excitation function is harmonic, the responses $w(x, t)$ and $\bar{w}(x, t)$ should be also harmonic. If there are other harmonic components, they must be due to non-linearities.

One can see from Eq. (13) that, if $M$ is too large, the maximum available force amplitude (i.e., $\left.F_{0} m / M\right)$ becomes too small for exciting a large structure. On the other hand, if a small structure (and hence a small $E I w^{\prime \prime \prime}(0, t)$ ) is to be tested, one should choose a large $M$ and hence a large $F_{0}$ can be used to make the $E I_{1}^{\prime \prime \prime}(0, t)$ relatively negligible. This is the reason we use a $768-\mathrm{g}$ fixture in this study of a small flexible beam. If the shaker controller DSC4-CE is used to monitor the base motion and to accordingly change the input voltage to the shaker to make $b(t)=B_{0} \sin \Omega t$, then we have

$$
m \ddot{\bar{v}}+c \dot{\bar{w}}+E I w^{i v}=m \Omega^{2} B_{0} \sin \Omega t .
$$

The free undamped linear mode shapes $\phi_{i}(x)$ of a cantilevered beam can be obtained from Eq. (10) to be

$$
\phi_{i}(x)=\cosh \beta_{i} x-\cos \beta_{i} x+\frac{\cos \beta_{i} L+\cosh \beta_{i} L}{\sin \beta_{i} L+\sinh \beta_{i} L}\left(\sin \beta_{i} x-\sinh \beta_{i} x\right) .
$$

For the first four modes, $\beta_{i} L=1.875104,4.694091,7.854757$, and 10.99554. Fig. 3 shows the first four mode shapes. We note that the maximum displacement of each mode is 2 at $x=L$. The instant mass center of each mode can be calculated to be at $(x, z)=$ $(L / 2,0.7830),(L / 2,0.4339),(L / 2,0.2544)$, and $(L / 2,0.1819)$. We note that the mass center gets close to the equilibrium position $(z=0)$ when the mode number increases.

We note that, because of the lumped mass $M$ of the movable part and the distributed structural weight $m g$, the actual natural frequencies and mode shapes may deviate from the ones shown in Eq. (15). To include the actual influences of the movable mass and the structural weight we add

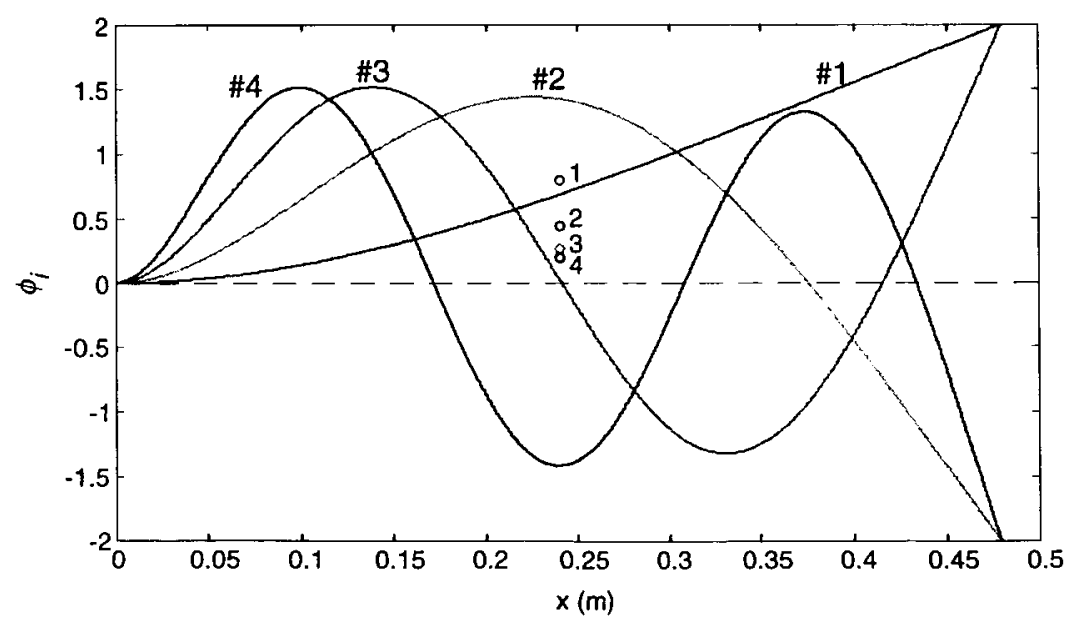

Fig. 3. The first four linear mode shapes and their instantaneous mass centers. 
the influence of structural weight to Eq. (13) to obtain that [9]

$$
m \ddot{\bar{w}}+c \dot{\bar{w}}+E I w^{, i}=\frac{m}{M}\left[E I w^{\prime \prime \prime}(0, t)-F_{0} \sin \Omega t\right]+\left[w^{\prime \prime}(x-L)+w^{\prime}\right] m g .
$$

From the linear mode shapes and their instantaneous mass centers shown in Fig. 3, we know that the gravity will slow down the restoration of bending if the beam is upward (Fig. 1), and the gravity will accelerate the restoration of bending if the beam is downward. Hence, the natural frequencies of the upward beam are expected to be less than those of the downward beam. Because the instantaneous mass center of a high-frequency mode is close to the equilibrium position, the influence of gravity on its natural frequency is expected to be small. If the mode shapes are assumed to be the same as those in Eq. (15), one can substitute the mode shapes in Eq. (15) into Eq. (16) (without $F_{0} \sin \Omega t$ and $c \dot{\bar{v}}$ ) and use the Galerkin method to estimate the natural frequencies $\omega_{i}$ as

$$
\omega_{i}^{2}=\beta_{i}^{4} \frac{E I}{m}-\frac{E I}{M} \frac{\phi_{i}^{\prime \prime \prime}(0) \int_{0}^{L} \phi_{i} \mathrm{~d} x}{\int_{0}^{L} \phi_{i}^{2} \mathrm{~d} x}-g \frac{\int_{0}^{L}\left[\phi_{i}^{\prime \prime}(x-L)+\phi^{\prime}\right] \phi \mathrm{d} x}{\int_{0}^{L} \phi_{i}^{2} \mathrm{~d} x} .
$$

It can be shown that $\int_{0}^{L} \phi_{i}^{2} \mathrm{~d} x=L$. If $M \rightarrow \infty$ and the gravity is neglected, the first four $\omega_{i}(=$ $\beta_{i}^{2} \sqrt{E I / m}$ ) are obtained to be $1.6964,10.6310,29.7671$, and $58.3316 \mathrm{~Hz}$. If only the influence of $M(=0.968 \mathrm{~kg})$ is considered, $\omega_{i}$ are obtained to be $1.7222,10.6810,29.8153$, and $58.3800 \mathrm{~Hz}$. One can see that the $M$ increases the natural frequencies by $1.5 \%, 0.5 \%, 0.2 \%$, and $0.1 \%$, respectively. Hence, the influence of the moving part on the vibration characteristics is expected to be small. If only the gravity is considered, $\omega_{i}$ of the upward beam are obtained to be $1.4365,10.4181,29.5491$, and $58.1026 \mathrm{~Hz}$, and $\omega_{i}$ of the downward beam are obtained to be $1.9214,10.8397,29.9835$, and $58.5598 \mathrm{~Hz}$. One can see that the gravity changes the natural frequencies of the downward (upward) beam by $13.3 \%(-15.3 \%), 2.5 \%(-2.0 \%), 0.7 \%(-0.7 \%)$, and $0.4 \%(-0.4 \%)$, respectively. In other words, the gravity will increase the first four natural frequencies from the upward beam to the downward beam by $28.6 \%, 4.5 \%, 1.4 \%$, and $0.8 \%$, respectively. Hence, the influence of gravity is more significant than that of the moving part on the vibration characteristics of the beam. Moreover, we note that both the moving mass and gravity do not have significant influences on high-frequency modes.

\section{Experimental and numerical results}

We used a $0-200 \mathrm{~Hz}$ periodic chirp signal for excitation and the PSV-200 scanning laser vibrometer to obtain the frequency response functions (FRFs) of 51 equally spaced points on the beam shown in Fig. 1. The measurement time was about $50 \mathrm{~min}$. Because 6400 FFT lines (i.e., only $200 i / 6400 \mathrm{~Hz}(i=1,2, \ldots, 6400)$ harmonics are included in the chirp signal, and the FRF will be calculated only at these frequencies) were used in the measurement, the frequency resolution is $0.03125(=200 / 6400) \mathrm{Hz}$. Fig. 4 shows the averaged FRF of the 51 FRFs of the upward beam. We note that the peaks in Fig. 4 are sharp and do not show the influence of damping because the linear scale is used in order to show the first peak. The first six peaks in Fig. 4 correspond to the first six natural frequencies shown in Table 1. From the averaged FRF of the 51 FRFs of the downward beam, the first six natural frequencies were also obtained and 


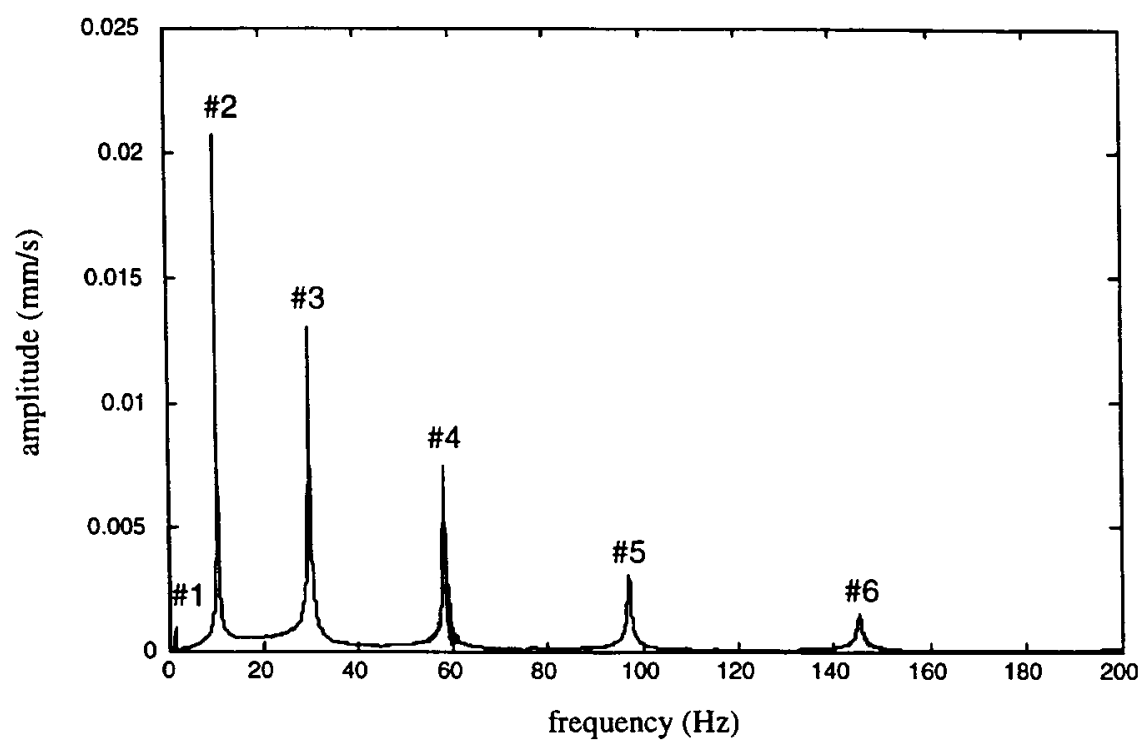

Fig. 4. The averaged frequency response function and the first six natural frequencies.

Table 1

Natural frequencies from the averaged FRF obtained using a periodic chirp excitation

\begin{tabular}{|c|c|c|c|c|c|c|}
\hline & (1) $(\mathrm{Hz})$ & $\omega_{2}(\mathrm{~Hz})$ & $\omega_{3}(\mathrm{~Hz})$ & $\omega_{4}(\mathrm{~Hz})$ & $\omega_{5}(\mathrm{~Hz})$ & $(1)_{6}(\mathrm{~Hz})$ \\
\hline Upward & 1.406 & $\begin{array}{l}10.438 \\
\left(\omega_{3} / 3+0.532\right) \\
\left(\omega_{4} / 6+0.725\right)\end{array}$ & $\begin{array}{l}29.719 \\
\left(3 \omega_{2}-1.595\right) \\
\left(0_{4} / 2+0.579\right)\end{array}$ & $\begin{array}{l}58.281 \\
\left(6 o_{2}-4.347\right) \\
\left(2 o_{3}-1.157\right)\end{array}$ & 97.125 & 145.438 \\
\hline Downward & 1.875 & $\begin{array}{l}10.875 \\
\left(\omega_{3} / 3+0.823\right) \\
\left(\omega_{4} / 6+1.083\right)\end{array}$ & $\begin{array}{l}30.156 \\
\left(3 \omega_{2}-2.469\right) \\
\left(\omega_{4} / 2+0.781\right)\end{array}$ & $\begin{array}{l}58.750 \\
\left(6 \omega_{2}-6.500\right) \\
\left(2 \omega_{3}-1.562\right)\end{array}$ & 97.469 & 145.875 \\
\hline $\begin{array}{l}\text { Increase } \\
(\%)\end{array}$ & 33.36 & 4.19 & 1.47 & 0.80 & 0.35 & 0.30 \\
\hline
\end{tabular}

shown in Table 1. It is obvious that, when the beam is put downward, the gravitational load on the beam makes the beam stiffer and increases natural frequencies. We note that the experimental percentage increases of the first four natural frequencies from the upward beam to the downward beam are very close to the numerical predictions $(28.6 \%, 4.5 \%, 1.4 \%$, and $0.8 \%)$ obtained using Eq. (17). However, gravity does not have significant influences on natural frequencies of higher modes. One can see that the second, third, and fourth natural frequencies are close to 10, 30, and $60 \mathrm{~Hz}$, respectively. Hence, cubic and quadratic non-linearities may cause 1:3 and/or $1: 2$ internal resonances (i.e., $3 \omega_{i} \approx \omega_{j}$ and/or $2 \omega_{i} \approx \omega_{j}$ ) among these three modes [10].

Then we performed time-domain acquisitions with the beam being excited at a chosen frequency, and 1024 velocity samples at each of the 51 points on the beam and one point on the 
fixture base were recorded using a sampling frequency of $512 \mathrm{~Hz}$. If the beam vibration is periodic and has a period $T$ and the recording at each location is controlled by triggering to begin at $n T$ ( $n$ is an integer) after the beginning time of the previous recording, the velocity profile at $t=t_{k}$ can be obtained by connecting the measured velocities of the 51 locations at $t_{k}$. To separate the contribution of each linear mode from the velocity profile at $t=t_{k}$ we assume

$$
\dot{w}\left(x, t_{k}\right)=\dot{b}\left(t_{k}\right)+\sum_{i=1}^{4} a_{i}\left(t_{k}\right) \phi_{i}(x),
$$

where $\dot{b}$ is the experimental base velocity and $a_{i}$ denotes the modal velocity of the $i$ th linear mode. To obtain the values of $a_{i}\left(t_{k}\right)$ by least-squares fitting we define a spatial-domain error function $E x$ as

$$
E x \equiv \sum_{m=1}^{51}\left[\dot{\omega}\left(x_{m}, t_{k}\right)-\dot{\hat{w}}\left(x_{m}, t_{k}\right)\right]^{2}
$$

where $\dot{\hat{w}}$ are experimental data. The equations for determining $a_{i}\left(t_{k}\right)$ are given by

$$
\frac{\partial E x}{\partial a_{i}}=\sum_{m=1}^{51} 2\left[\dot{u}\left(x_{m}, t_{k}\right)-\dot{\hat{\imath}}\left(x_{m}, t_{k}\right)\right] \phi_{i}\left(x_{m}\right)=0, \quad i=1,2,3,4 .
$$

The standard deviation $S D$ of the fitted velocity profile at each instant can be calculated as

$$
S D \equiv \sqrt{\frac{E_{x}}{N}}
$$

where $N$ denotes the number of measurement points, which is 51 in this study. Because the scanning laser vibrometer uses the known excitation frequency and a trigger to determine the beginning time for recording at each location on the beam, the recorded velocities of the 52 locations (including the point on the fixture) will be in phase if the velocities contain only harmonics of the excitation frequency $\Omega$ and its integral multiples (i.e., $n \Omega$ ). If the motion contains non-periodic components or periodic components with frequencies different from $n \Omega$, the obtained velocity profiles may not be the actual ones because artificial phase differences may be introduced by the data acquisition. However, one can check the velocities of the point at $x=x_{m}$ to see whether its curve-fitted velocities $i\left(x_{m}, t_{k}\right), k=1, \ldots, 1024$ in Eq. (18) match with its experimental velocities $\dot{\hat{w}}\left(x_{m}, t_{k}\right)$, and it can be quantified using the following time-domain error function $E t$ :

$$
E t \equiv \sum_{k=1}^{1024}\left[\dot{\mathfrak{w}}\left(x_{m}, t_{k}\right)-\dot{\hat{w}}\left(x_{m}, t_{k}\right)\right]^{2}
$$

Fig. 5 shows the operational deflection shapes captured using a SONY DSCP1 digital camera when the base was excited at the second, third, and fourth natural frequencies, respectively. Figs. 6(a)-(d) show 50 (25 light lines and then 25 dark lines) consecutive experimental velocity profiles of the vibration shown in Fig. 5(a), the velocity profiles curve-fitted using Eq. (18) and numerical velocity profiles obtained using the multiple shooting method, the modal velocity $a_{i}$, and the fitting error, respectively. The roughness of experimental velocity profiles shown in Fig. 6(a) is due to spectral noise caused by the small longitudinal displacement and rotation of the 


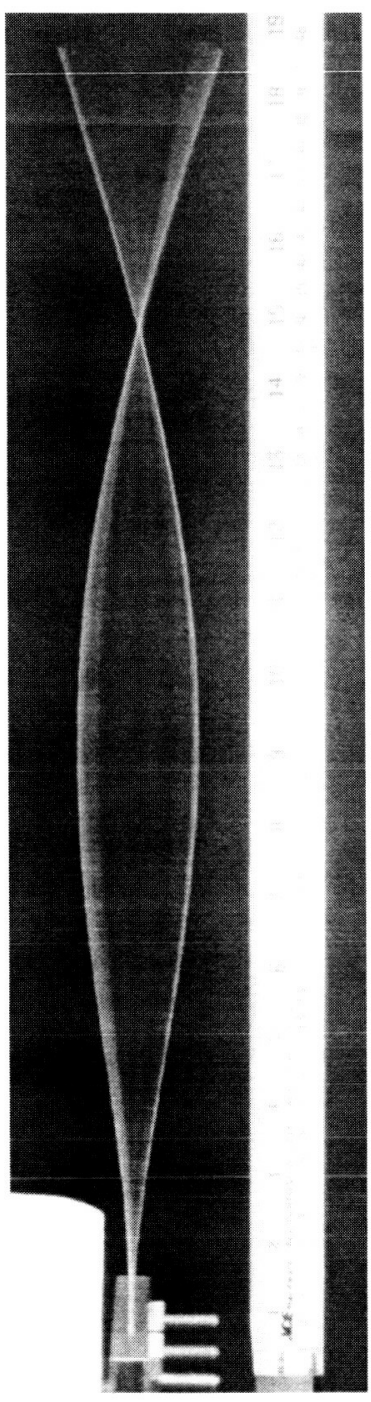

(a)

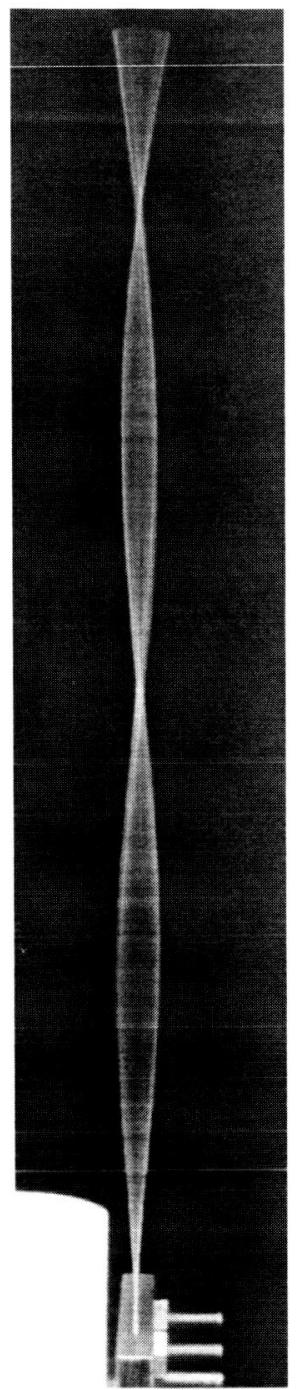

(b)

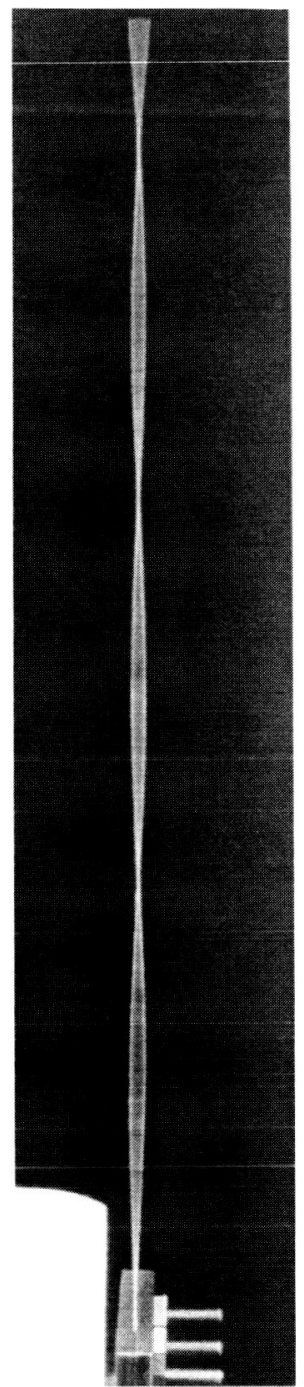

(c)

Fig. 5. The operational deflection shape when the beam is subjected a harmonic base-excitation at: (a) $\Omega=\omega_{2}=$ $10.438 \mathrm{~Hz}$ with $\dot{b}_{\max }=0.0723 \mathrm{~m} / \mathrm{s}$, (b) $\Omega=\omega_{3}=29.719 \mathrm{~Hz}$ with $\dot{b}_{\max }=0.0884 \mathrm{~m} / \mathrm{s}$, and (c) $\Omega=\omega_{4}=58.281 \mathrm{~Hz}$ with $\dot{b}_{\max }=0.0422 \mathrm{~m} / \mathrm{s}$.

beam, and it is more serious when the deflection is large and hence the velocity is close to zero. The DSC4-CE controller was set to control the base velocity amplitude to be $0.07 \mathrm{~m} / \mathrm{s}$ and the actual amplitude was $0.0723 \mathrm{~m} / \mathrm{s}$. This $3 \%$ error is typical for all the experiments reported in this paper, and the controlled base motion was found to be pretty close to a harmonic curve. The intersection of velocity profiles within the envelope in Fig. 6(b) indicates that the motion consists of two or more modes. The two starred lines in Fig. 6(b) are obtained using the multiple shooting method [5,8] to solve Eqs. (1), (4), and (5). Because no damping is included in Eq. (1), the 
predicted amplitude is larger than the actual one. Moreover, in order to prevent the laser beam from missing the beam tip (see Point $c$ in Fig. 2), the last measurement point was set a little bit away from the beam tip and hence the theoretical node does not coincide with the experimental one. We also used the multiple shooting method to solve Eqs. (8) and (9) for theoretical velocity profiles, and the solutions are larger than those theoretical ones in Fig. 6 (b) by $1.2 \%$ or less. In other words, although the amplitude is rather large (see Fig. 5(a)), including non-linearities up to cubic terms is accurate enough for this study. However, because Eq. (8) is not geometrically exact and its solution converges slowly in the multiple shooting process, all the theoretical velocity profiles presented were obtained using Eqs. (1)-(5). Fig. 6(c) shows that the velocity profiles mainly consist of the first and second linear modes and the contributions of third and fourth linear modes are negligible. Moreover, both non-trivial modes are almost harmonic, vibrate at the excitation frequency, and have a constant phase difference $\boldsymbol{x}$.

The $\phi_{i}(x)$ shown in Eq. (15) are called linear normal mode shapes because they are derived from the linear governing Eq. (10) and because all points on the beam pass the equilibrium position $(\bar{w}(x, t)=0)$ at the same time when the vibration is $\bar{w}(x, t)=\phi_{i}(x) \sin \omega_{i} t$. The two starred lines shown in Fig. 6(b) represent a non-linear normal mode shape because it is obtained by assuming $\bar{w}(x, t)=\Phi(x) \sin \Omega t$ in Eq. (4). If two (or more) arbitrary, linear normal modes vibrate at the same frequency $\Omega$ with a constant phase difference $\alpha$, they can be combined into one mode (in order to reduce the number of modes and computation efforts) as

$$
\begin{aligned}
& \bar{w}=\hat{\phi}_{2}(x) \sin \Omega t+\hat{\phi}_{1}(x) \sin (\Omega t+x)=\Phi(x) \sin (\Omega t+\bar{x}), \\
& \hat{\phi}_{1} \equiv \frac{a_{1 \max }}{\Omega} \phi_{1}, \quad \hat{\phi}_{2} \equiv \frac{a_{2 \max }}{\Omega} \phi_{2}, \\
& \Phi(x) \equiv \sqrt{\hat{\phi}_{2}^{2}+2 \hat{\phi}_{1} \hat{\phi}_{2} \cos \alpha+\hat{\phi}_{1}^{2}}, \quad \bar{x} \equiv \tan ^{-1} \frac{\hat{\phi}_{1} \sin \alpha}{\hat{\phi}_{2}+\hat{\phi}_{1} \cos \alpha} .
\end{aligned}
$$

Eq. (23) shows that $\bar{\alpha}$ is a constant (i.c., zero) only if $\alpha=0^{\circ}$ or $180^{\circ}$. If $\alpha \neq 0^{\circ}$ or $180^{\circ}, \bar{\alpha}$ is a function of $x$ and it is called a complex mode, i.e., they cannot be combined into a normal mode. Because Fig. 6(c) shows that the phase difference $\alpha$ between $a_{1}(t)$ and $a_{2}(t)$ is not $0^{\circ}$ or $180^{\circ}$, it is a complex mode. Close examination of Fig. 6(a) also shows that the maximum velocity profile (i.e., minimum deflection) between the clamped end and the node does not occur when the velocity profile between the node and the beam tip is maximum, and hence it is a complex mode. On the other hand, using the multiple shooting solution $\vec{w}$ obtained from Eqs. (1) $-(5)$ to estimate the maximum velocity profile as $\Omega(b+\bar{w})$ implies the assumption that $\bar{\alpha}$ is constant and it is a normal mode. This is another factor that causes the difference between the theoretical and experimental maximum velocity profiles in Fig. 6(b). Fig. 6(d) shows that, when the velocity is zero (i.e., maximum deffection), the maximum curve fitting error occurs because of spectral noise. In Fig. $6(\mathrm{~d}), W_{m}$ denotes the maximum value of $\dot{\hat{w}}$. When the velocity is the maximum or minimum, the error is the minimum because it has a large signal-to-noise ratio and the beam is almost flat.

Figs. 7(a)-(c) show 58 (29 light lines and then 29 dark lines) consecutive experimental velocity profiles of the beam excited at $9.0 \mathrm{~Hz}$, the curve-fitted velocity profiles, and the modal velocities $a_{i}$, respectively. Because the vibration amplitude in Fig. 7(b) is smaller than that in Fig. 6(b), the last measurement point (Point $\mathrm{c}$ in Fig. 2) was set closer to the beam tip and hence the theoretical node almost coincides with the experimental one. Because the first mode also vibrates at the excitation 

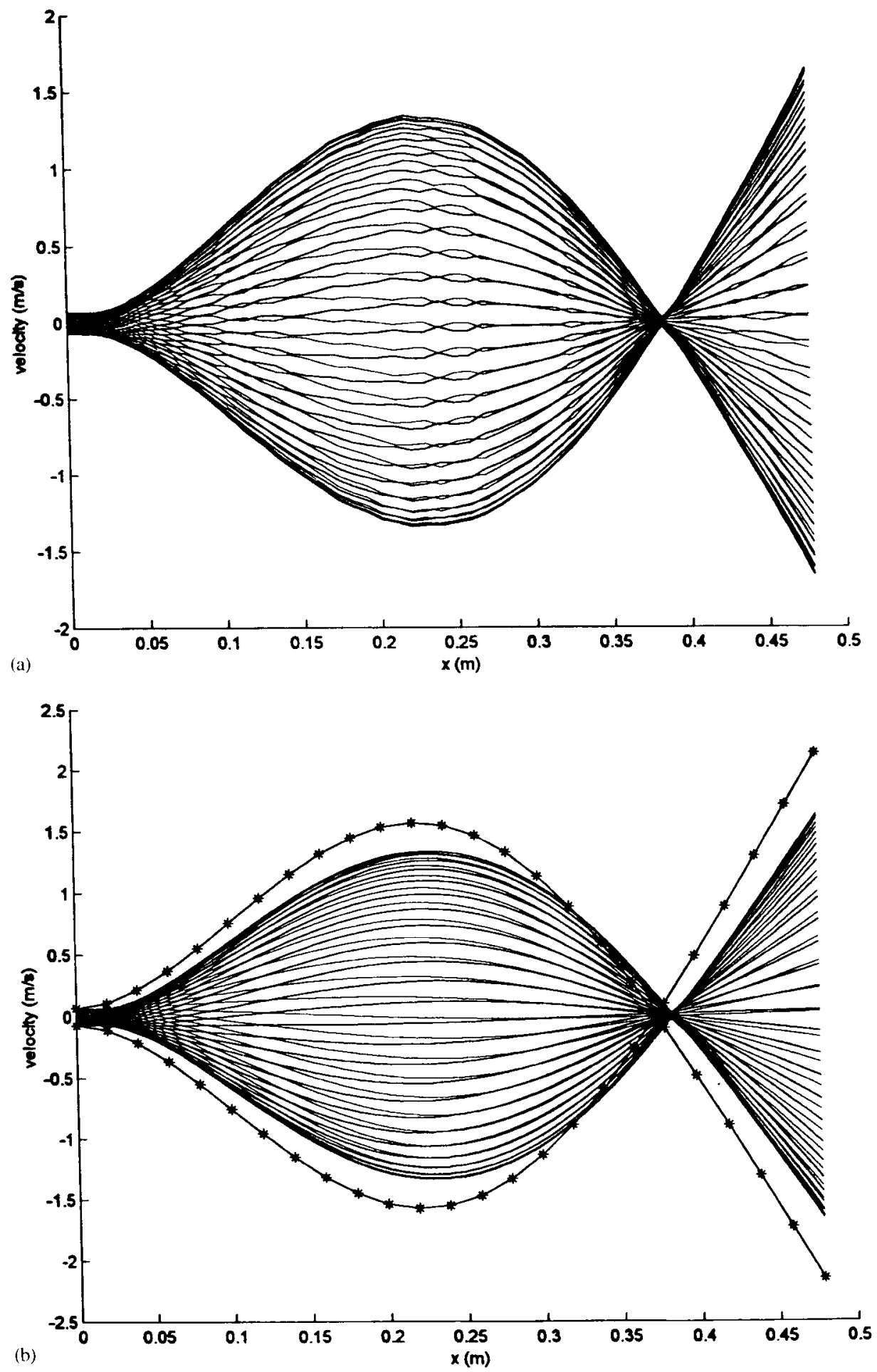

Fig. 6. 50 velocity profiles at consecutive time steps when $\Omega=10.438 \mathrm{~Hz}$ and $\dot{b}_{m u x}=0.0723 \mathrm{~m} / \mathrm{s}$ : (a) experimental data, (b) curve-fitted data and numerical prediction (stars), (c) modal velocities, and (d) standard deviation (SD) of the curvefitting. 

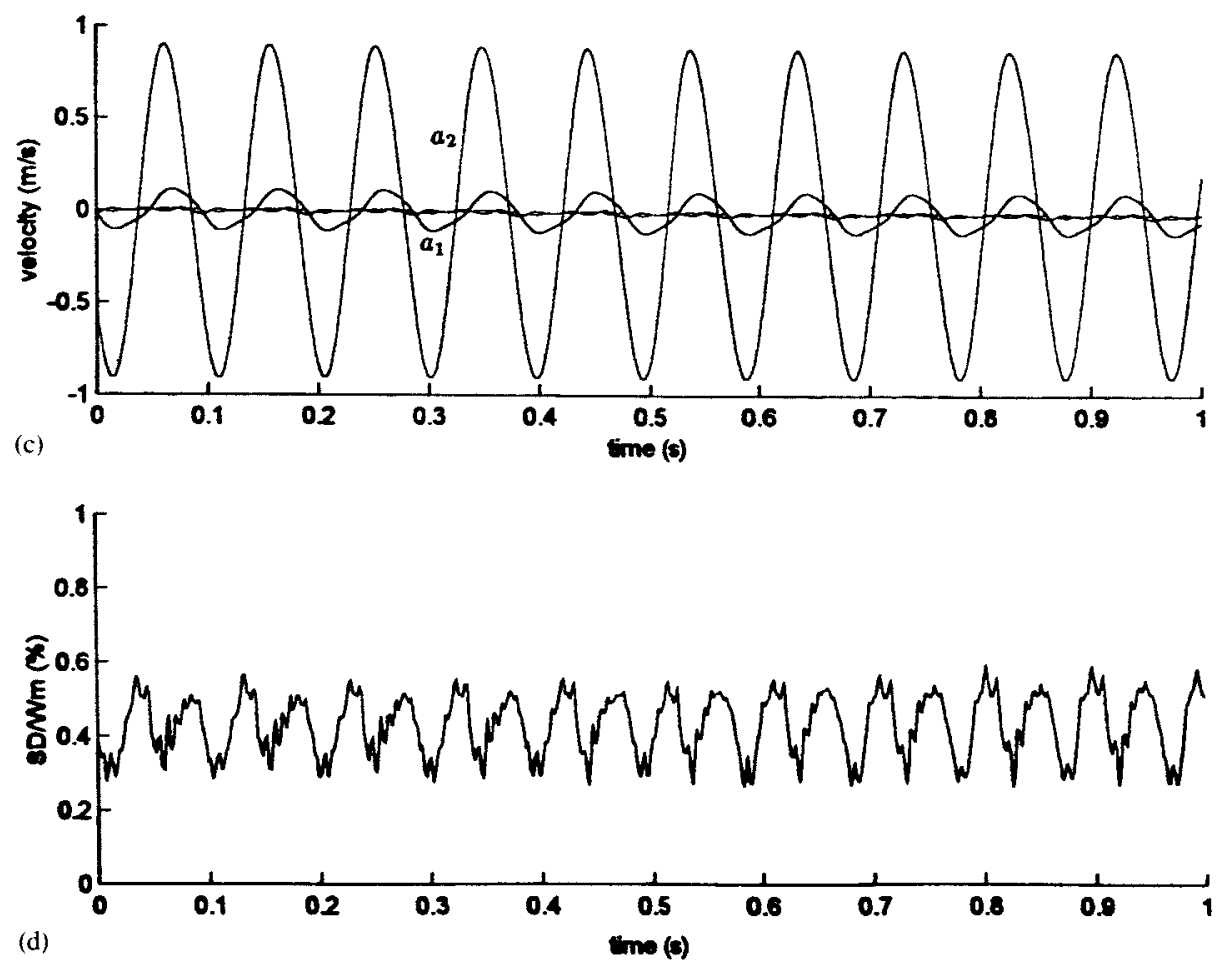

Fig. 6 (continued).

frequency and has a $180^{\circ}$ phase with respect to the second mode shape, it is a non-linear normal mode and the non-linear mode shape $\Phi(x)$ can be written as

$$
\Phi(x)=\frac{a_{2 \max }}{\Omega} \phi_{2}(x)-\frac{a_{1 \max }}{\Omega} \phi_{1}(x) .
$$

Figs. 8(a)-(c) show 44 (22 light lines and then 22 dark lines) consecutive experimental velocity profiles of the beam excited at $12.0 \mathrm{~Hz}$, the curve-fitted velocity profiles, and the modal velocities $a_{i}$, respectively. The theoretical velocity profiles in Figs. 7(b) and 8(b) agree well with the experimental ones because the excitation frequencies are away from the natural frequency and hence damping does not have significant influences. Because the first mode also vibrates at the excitation frequency and has a $0^{\circ}$ phase with respect to the second mode, it is a non-linear normal mode and the non-linear mode shape $\Phi(x)$ can be written as

$$
\Phi(x)=\frac{a_{2 \max }}{\Omega} \phi_{2}(x)+\frac{a_{1 \max }}{\Omega} \phi_{1}(x) .
$$

Figs. 6(c), 7(c), and 8(c) and Eqs. (23)-(25) show that, although the first two linear modes vibrate at the excitation frequency, the phase difference between them varies with the excitation frequency. Moreover, Figs. 6(b), 7(b), and 8(b) show that the theoretical non-linear mode shape (i.e., $(\dot{i}-\dot{b}) / \Omega$ ) changes with the excitation frequency $\Omega$. In other words, the first two linear modes move independently and cannot be combined into one non-linear normal mode. Hence, the 

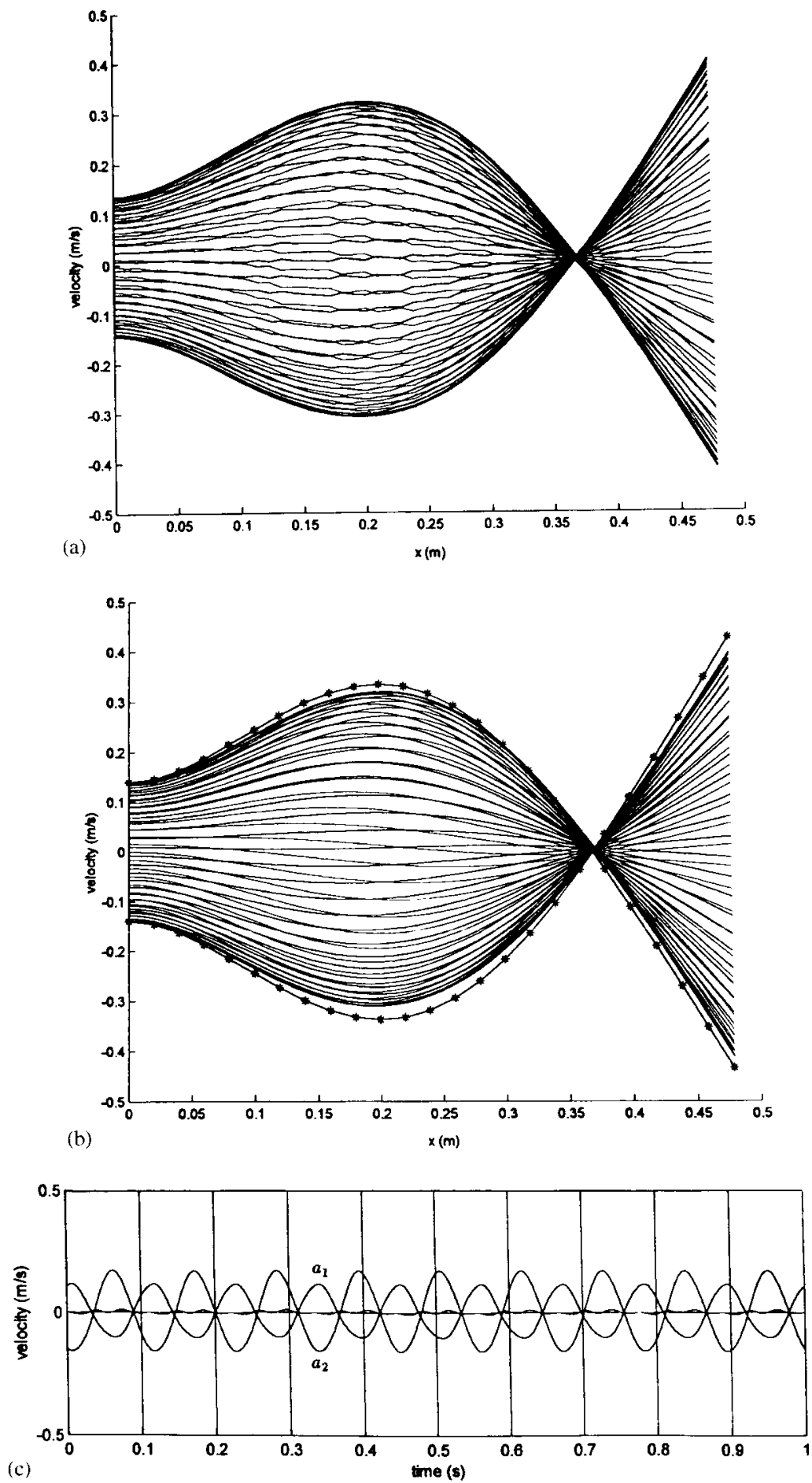

Fig. 7. 58 velocity profiles at consecutive time steps when $\Omega=9 \mathrm{~Hz}$ and $\dot{b}_{m a x}=0.1399 \mathrm{~m} / \mathrm{s}$ : (a) experimental data, (b) curve-fitted data and numerical prediction (stars), and (c) modal velocities. 

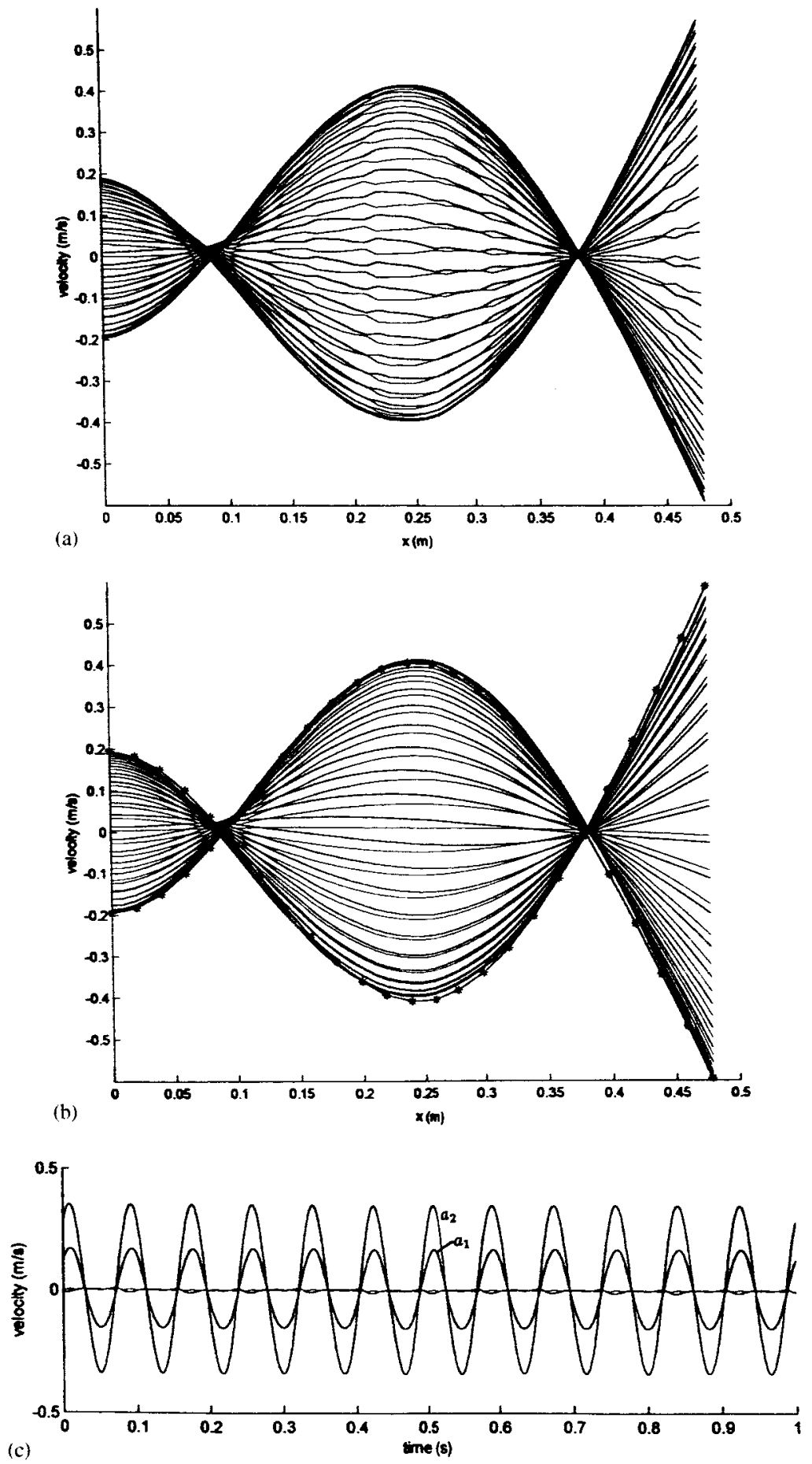

Fig. 8. 44 velocity profiles at consecutive time steps when $\Omega=12 \mathrm{~Hz}$ and $\dot{b}_{\text {max }}=0.1952 \mathrm{~m} / \mathrm{s}$ : (a) experimental data, (b) curve-fitted data and numerical prediction (stars), and (c) modal velocities. 

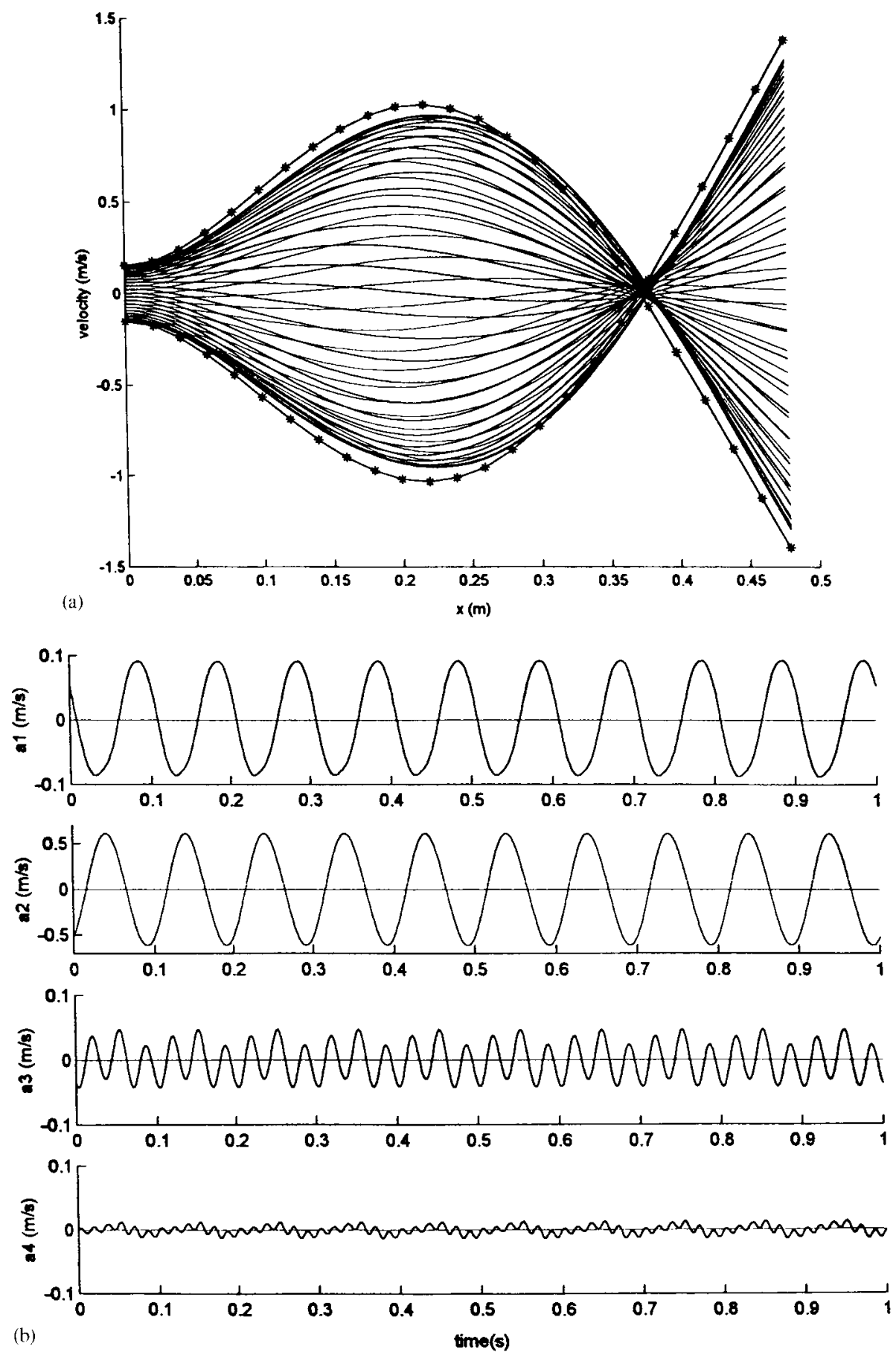

Fig. 9.52 velocity profiles at consecutive time steps when $\Omega=10 \mathrm{~Hz}$ and $\dot{b}_{\max }=0.1528 \mathrm{~m} / \mathrm{s}$ : (a) curve-fitted data and numerical prediction (stars), and (b) modal velocities. 

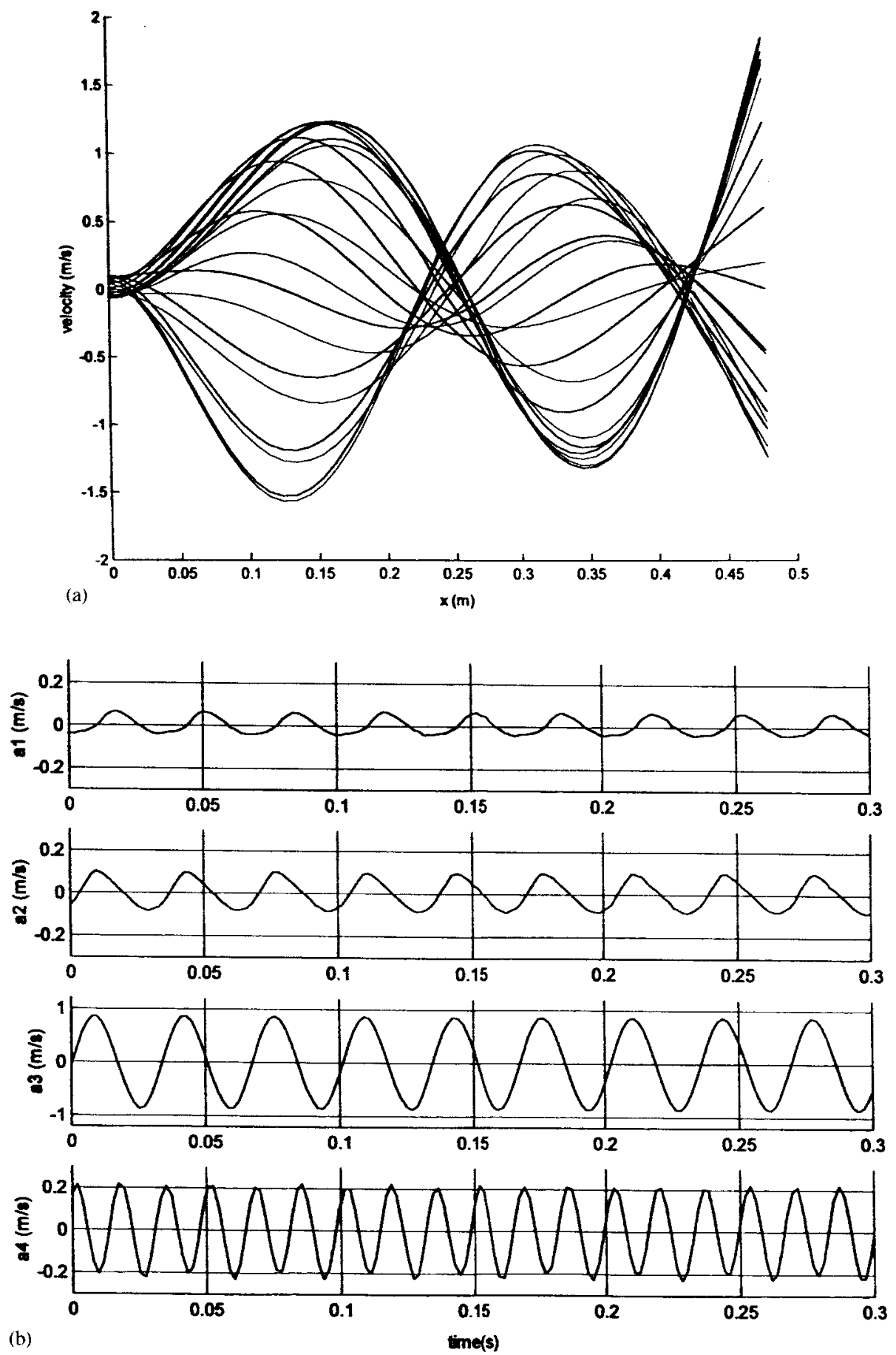

Fig. 10. 20 velocity profiles at consecutive time steps when $\Omega=29.719 \mathrm{~Hz}$ and $\dot{b}_{\max }=0.0884 \mathrm{~m} / \mathrm{s}$ : (a) curve-fitted data and (b) modal velocities. 

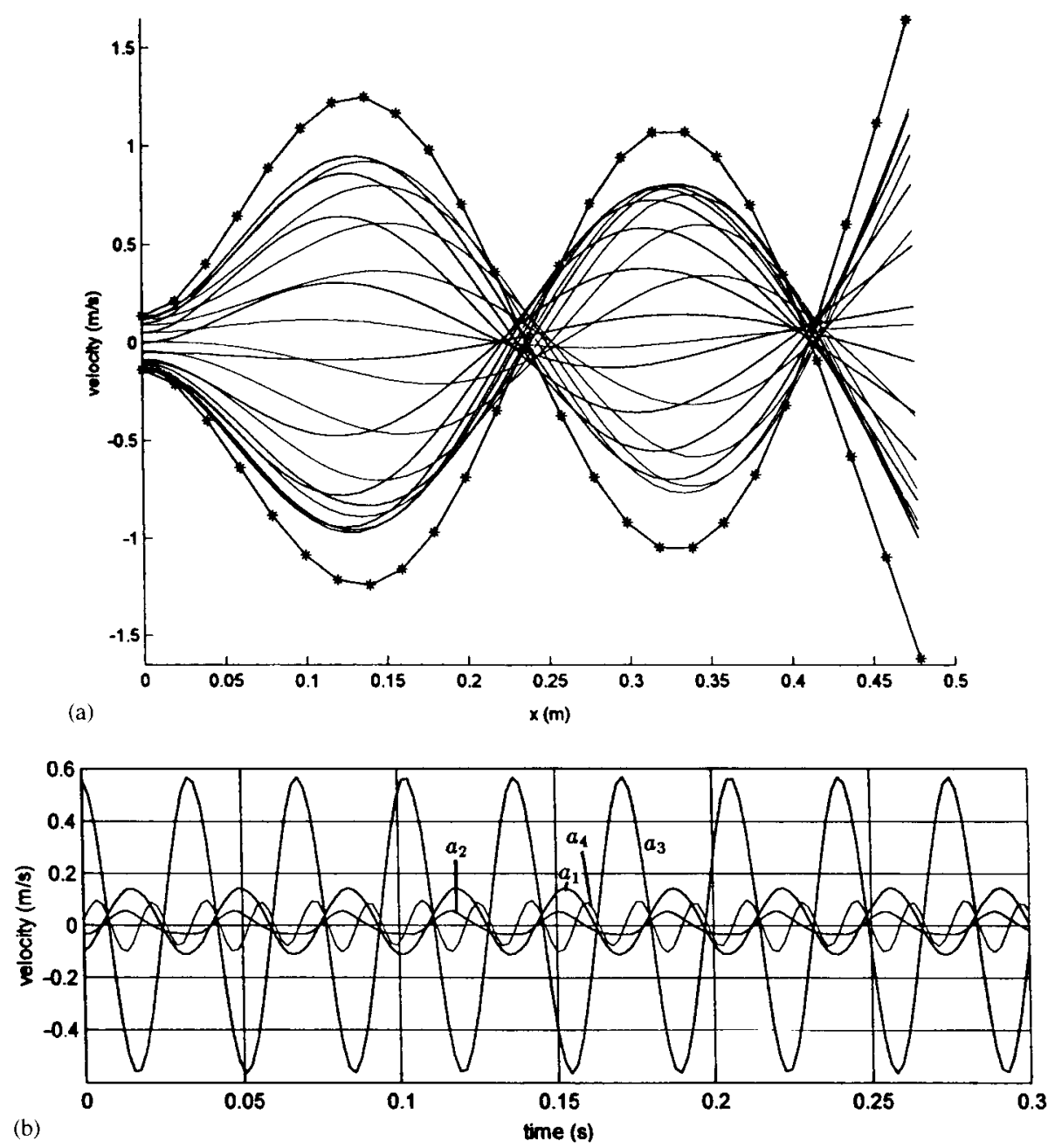

Fig. 11. 20 velocity profiles at consecutive time steps when $\Omega=29 \mathrm{~Hz}$ and $\dot{b}_{\max }=0.1371 \mathrm{~m} / \mathrm{s}$ : (a) curve-fitted data and numerical prediction (stars), and (b) modal velocities.

concept of using non-linear normal modes instead of linear modes to reduce the number of modes required in analyzing non-linear structural dynamics is questionable.

Figs. 9(a) and (b) show 52 (26 light lines and then 26 dark lines) consecutive curve-fitted velocity profiles of the beam excited at $10 \mathrm{~Hz}$, and the modal velocities $a_{i}$. Fig. 9(b) shows that the motion consists of the first four modes. The first mode vibrates at $10 \mathrm{~Hz}$ and is due to forced vibration. The third mode vibrates at $30 \mathrm{~Hz}$ and is caused by $1: 3$ external and/or internal resonances due to cubic non-linearities. The fourth mode vibrates at $60 \mathrm{~Hz}$ and is caused by 1:2:3 and/or $1: 6$ external and/or internal resonances.

Fig. 10 shows 20 (10 light lines and then 10 dark lines) consecutive curve-fitted velocity profiles of the beam excited at the third natural frequency $29.719 \mathrm{~Hz}$ (i.e., Fig. 5(b)), and the modal velocities $a_{i}$. The multiple shooting method was not able to find a converged operational 

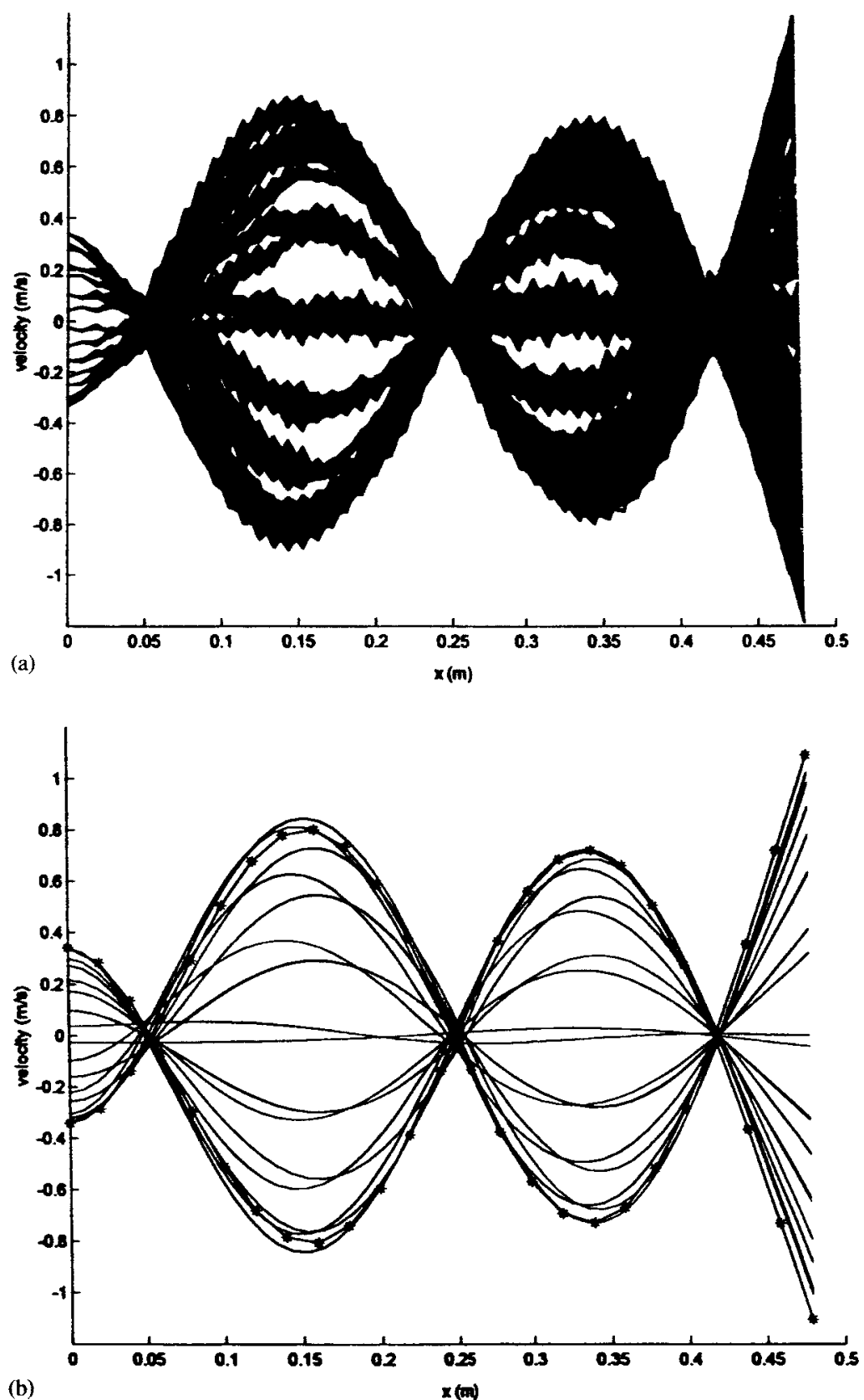

Fig. 12. 32 velocity profiles at consecutive time steps when $\Omega=32 \mathrm{~Hz}$ and $\dot{b}_{\max }=0.3414 \mathrm{~m} / \mathrm{s}$ : (a) experimental data (1024 profiles), (b) curve-fitted data and numerical prediction (stars), (c) modal velocities, (d) time trace of the 51st measurement point (at the beam tip), and (e) curve-fitted time trace of the 51 st measurement point. 

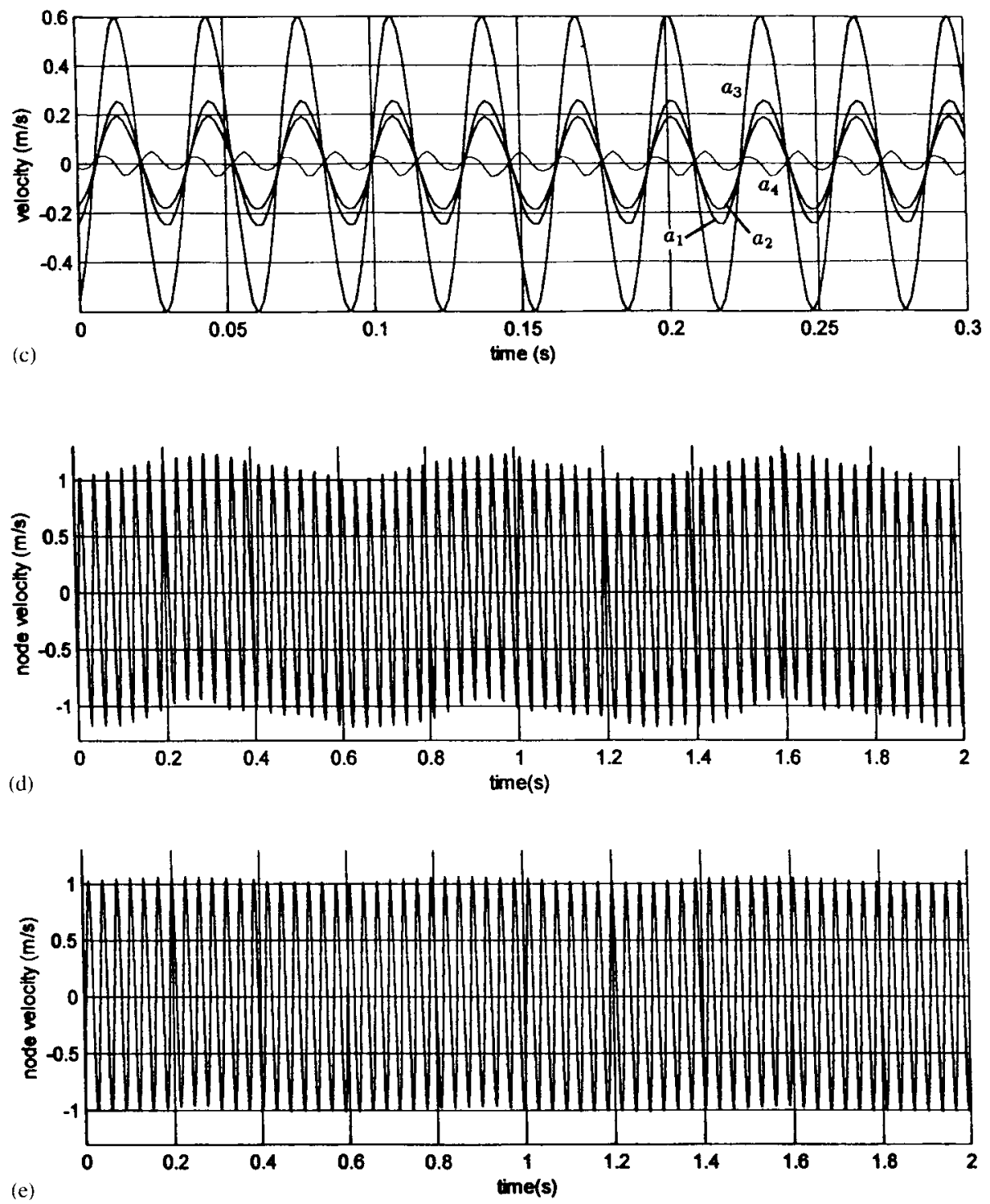

Fig. 12 (continued).

deflection shape for this case. Fig. 10(b) shows that the motion consists of the first four modes. The first three modes all vibrate at the excitation frequency and are due to forced vibration. The fourth mode vibrates at $2 \omega_{3}$ caused by $1: 2$ external and/or internal resonances due to quadratic non-linearities. Quadratic non-linearities may be due to initial imperfection (i.e., $k_{2} \neq 0$ ) or the beam being not really vertical. Fig. 10(b) also shows that every modal velocity arrives at zero at different times and it is why the nodes in Figs. 5(b) and 10(a) are not clear. Fig. 11 shows 20 (10 light lines and then 10 dark lines) consecutive curve-fitted velocity profiles of the beam excited at $29 \mathrm{~Hz}$, and the modal velocities $a_{i}$. Fig. 11(b) shows that the motion consists of the first four 

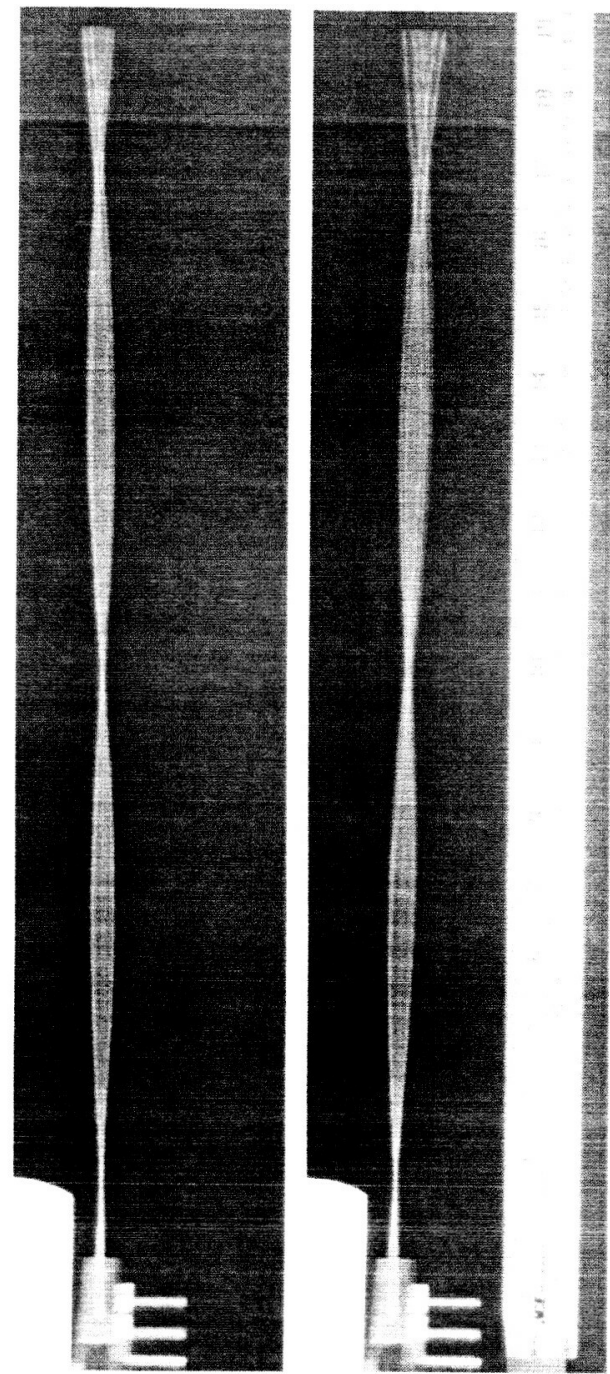

Fig. 13. The operational deflection shapes captured at two different times when $\Omega=32 \mathrm{~Hz}$ and $\dot{h}_{\text {max }}=0.3414 \mathrm{~m} / \mathrm{s}$.

modes. The first two modes vibrate at the excitation frequency and have a $180^{\circ}$ phase with respect to the third mode. The fourth mode vibrates at $58 \mathrm{~Hz}$ and has zero velocity when the first three modal velocities are close to zero.

Fig. 12(a) shows 1024 experimental velocity profiles of the beam excited at $32 \mathrm{~Hz}$, and Fig. 12(b) shows 32 consecutive curve-fitted velocity profiles. Fig. 12(c) shows that the motion consists of the first four modes. The first two modes vibrate at the excitation frequency and have a 0 phase with respect to the third mode (i.e.. the directly excited mode). The fourth mode vibrates at $64 \mathrm{~Hz}$ and has zero velocity when the first three modal velocities are close to zero. Because the sampling frequency $(512 \mathrm{~Hz})$ is $16(=512 / 32)$ times the excitation frequency $(32 \mathrm{~Hz})$ in this case. there should be only 16 different velocity profiles in Figs. 12(a) and (b) if the beam vibrates at 

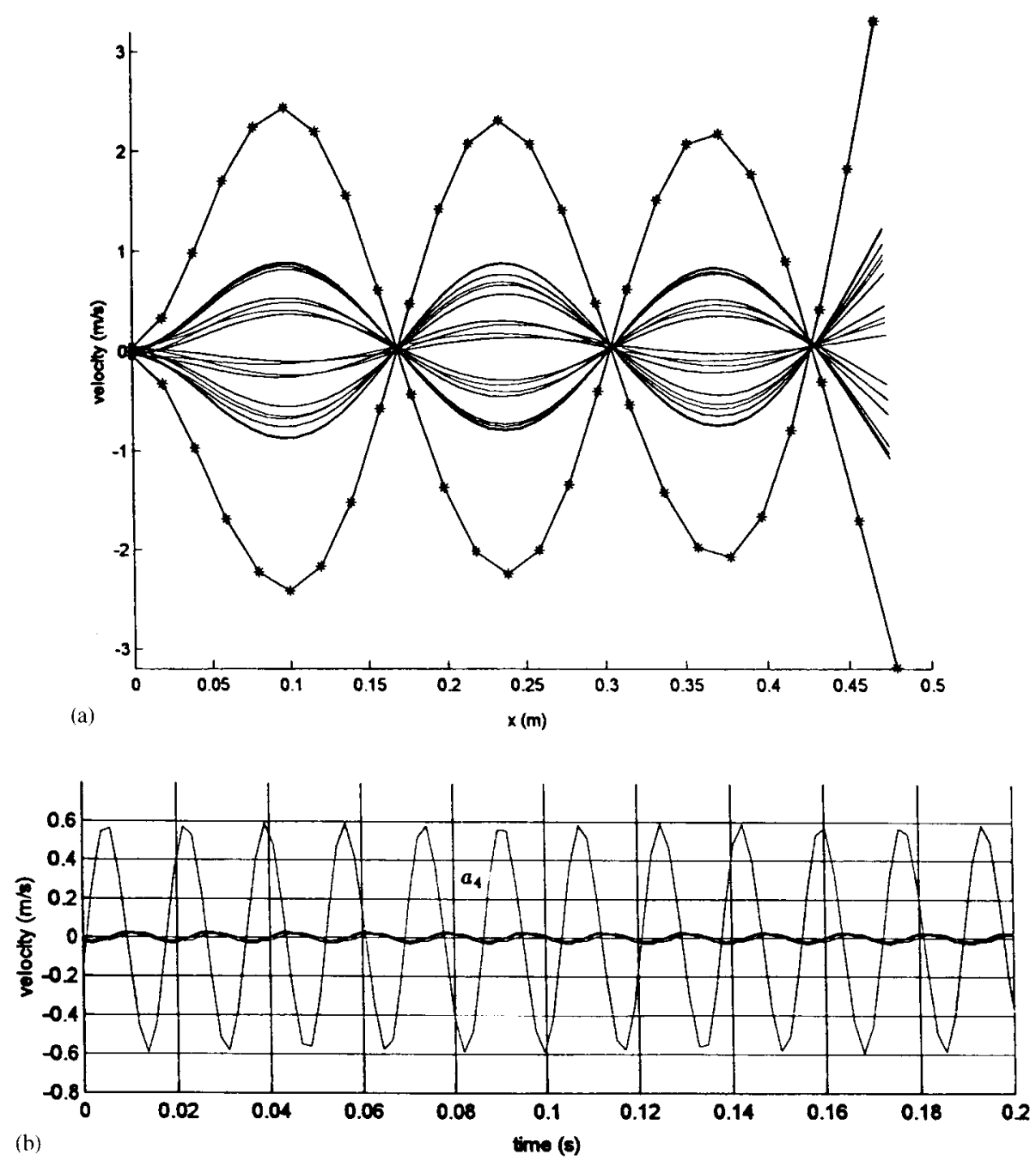

Fig. 14. 18 velocity profiles at consecutive time steps when $\Omega=58.281 \mathrm{~Hz}$ and $\dot{b}_{\max }=0.0422 \mathrm{~m} / \mathrm{s}$ : (a) curve-fitted data and numerical prediction (stars), and (b) modal velocities.

$32 n \mathrm{~Hz}$. Fig. 12(b) shows exactly 16 different curve-fitted velocity profiles although 32 consecutive velocity profiles are plotted. However, the non-overlapping profiles shown in Fig. 12(a) and the large fitting error (average $S D / W_{\max }=4.5 \%$, compared to $2.2 \%$ in Fig. 10 and $1.3 \%$ in Fig. 11 ) indicate that there are harmonic components different from $32 n \mathrm{~Hz}$. Figs. 12(d) and (e) show the experimental and curve-fitted time traces of the beam tip. It is apparent that the curve-fitting averages out the $1.406 \mathrm{~Hz}\left(=\omega_{1}\right)$ harmonic and the time-domain error function $E_{t}$ (see Eq. (22)) of each measurement point is higher than that in Figs. 10 and 11. Fig. 13 shows that the beam swung back and forth at $1.406 \mathrm{~Hz}$. Again Figs. 10(b), 11(b), and 12(c) show that linear modes cannot be combined into fewer non-linear normal modes and the existence of non-linear normal modes is questionable. 

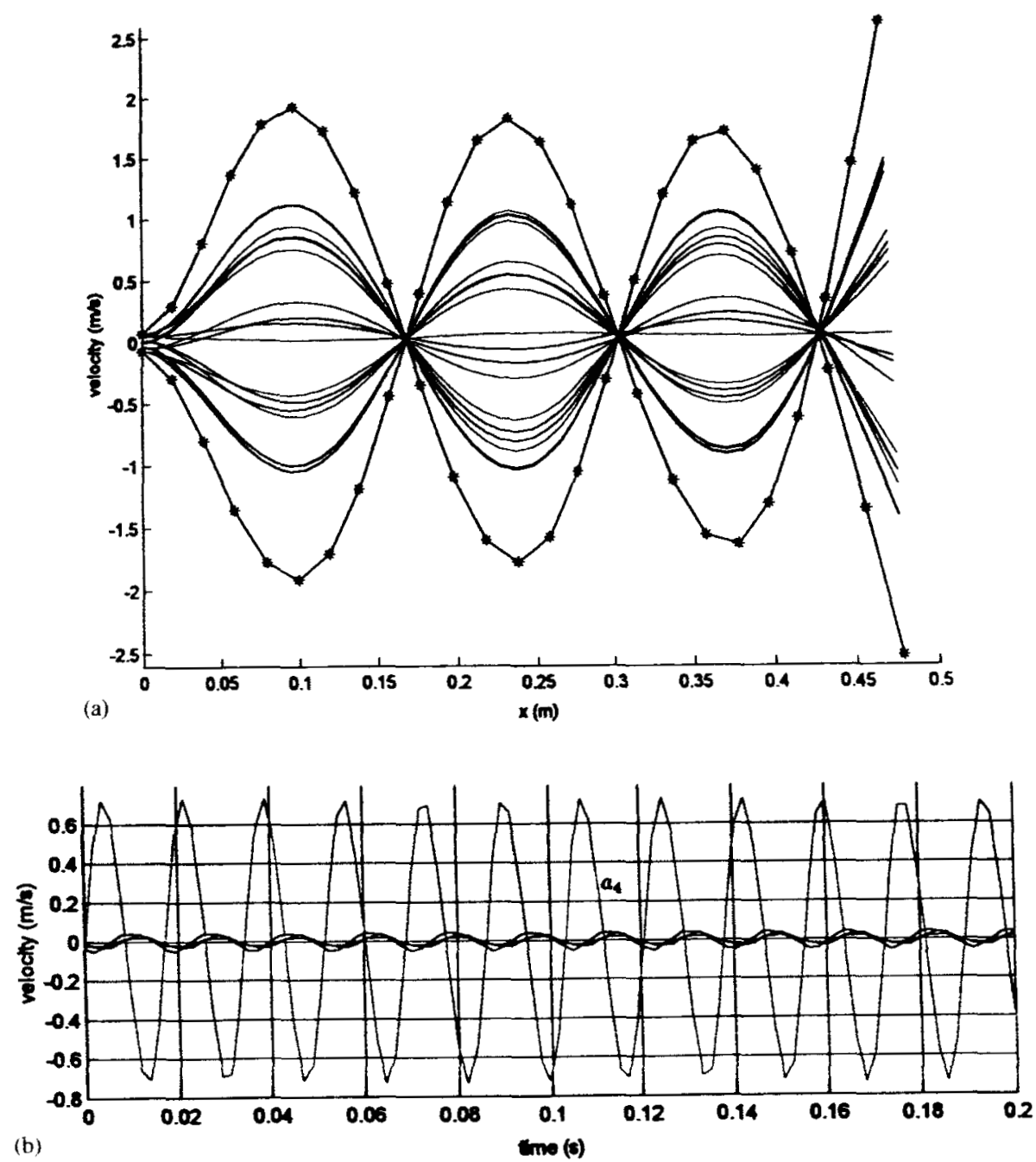

Fig. 15. 18 velocity profiles at consecutive time steps when $\Omega=58 \mathrm{~Hz}$ and $\dot{b}_{\max }=0.072 \mathrm{~m} / \mathrm{s}$ : (a) curve-fitted data and numerical prediction (stars), and (b) modal velocities.

Figs. 14(a) and (b) show 18 (9 light lines and then 9 dark lines) consecutive curve-fitted velocity profiles of the beam excited at the fourth natural frequency $58.281 \mathrm{~Hz}$ (i.e., Fig. 5(c)), and the modal velocities $a_{i}$, respectively. Again the theoretical velocity profiles obtained using the multiple shooting method are much larger than the actual ones because damping is not included in Eq. (1). Fig. 14(b) shows that the fourth mode dominates the motion, and the first three modes all vibrate at the excitation frequency and have a $90^{\circ}$ phase with respect to the fourth mode. Figs. 15(a) and (b) show 18 (9 light lines and then 9 dark lines) consecutive curve-fitted velocity profiles of the beam excited at $58 \mathrm{~Hz}$, and the modal velocities $a_{i}$, respectively. Fig. 15(b) shows that the fourth mode dominates the motion, and the first three modes all vibrate at the excitation frequency and 

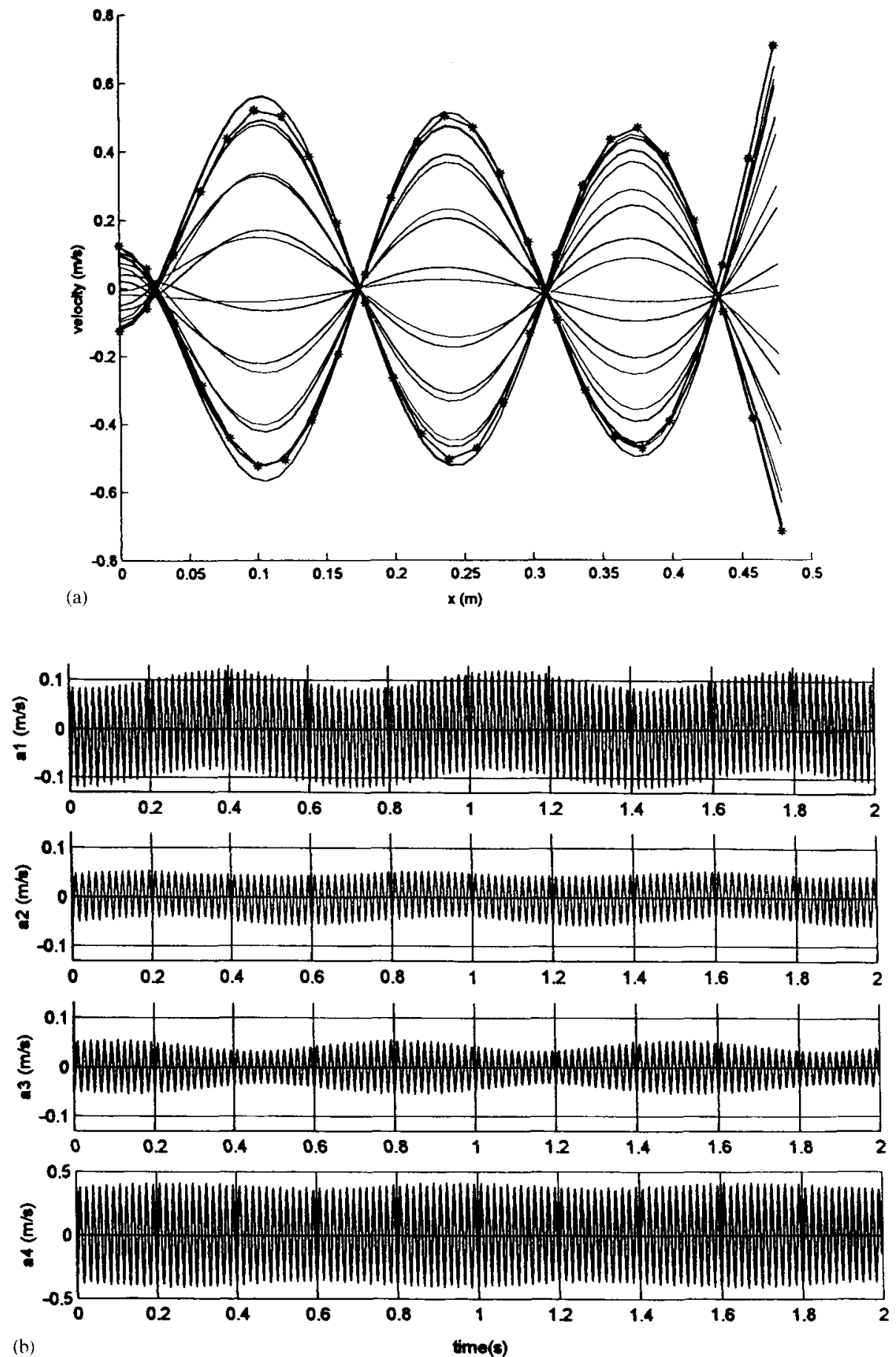

Fig. 16. 18 velocity profiles at consecutive time steps when $\Omega=60 \mathrm{~Hz}$ and $\dot{b}_{m a x}=0.1256 \mathrm{~m} / \mathrm{s}$ : (a) curve-fitted data and numerical prediction (stars), and (b) modal velocities. 


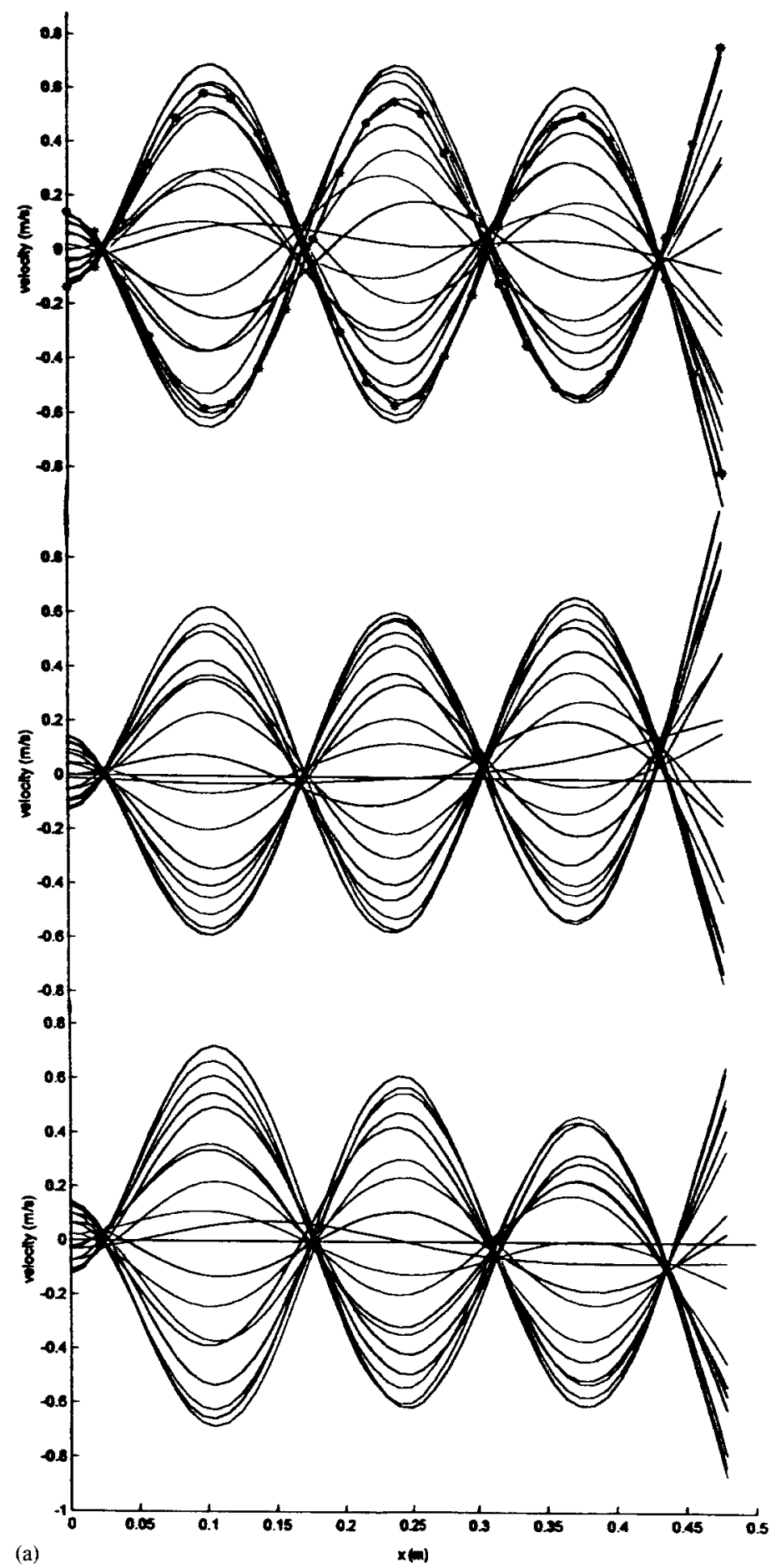

Fig. 17. 18 velocity profiles at consecutive time steps when $\Omega=60 \mathrm{~Hz}$ and $\dot{b}_{\max }=0.1395 \mathrm{~m} / \mathrm{s}$ : (a) curve-fitted data and numerical prediction (stars). and (b) modal velocities. 

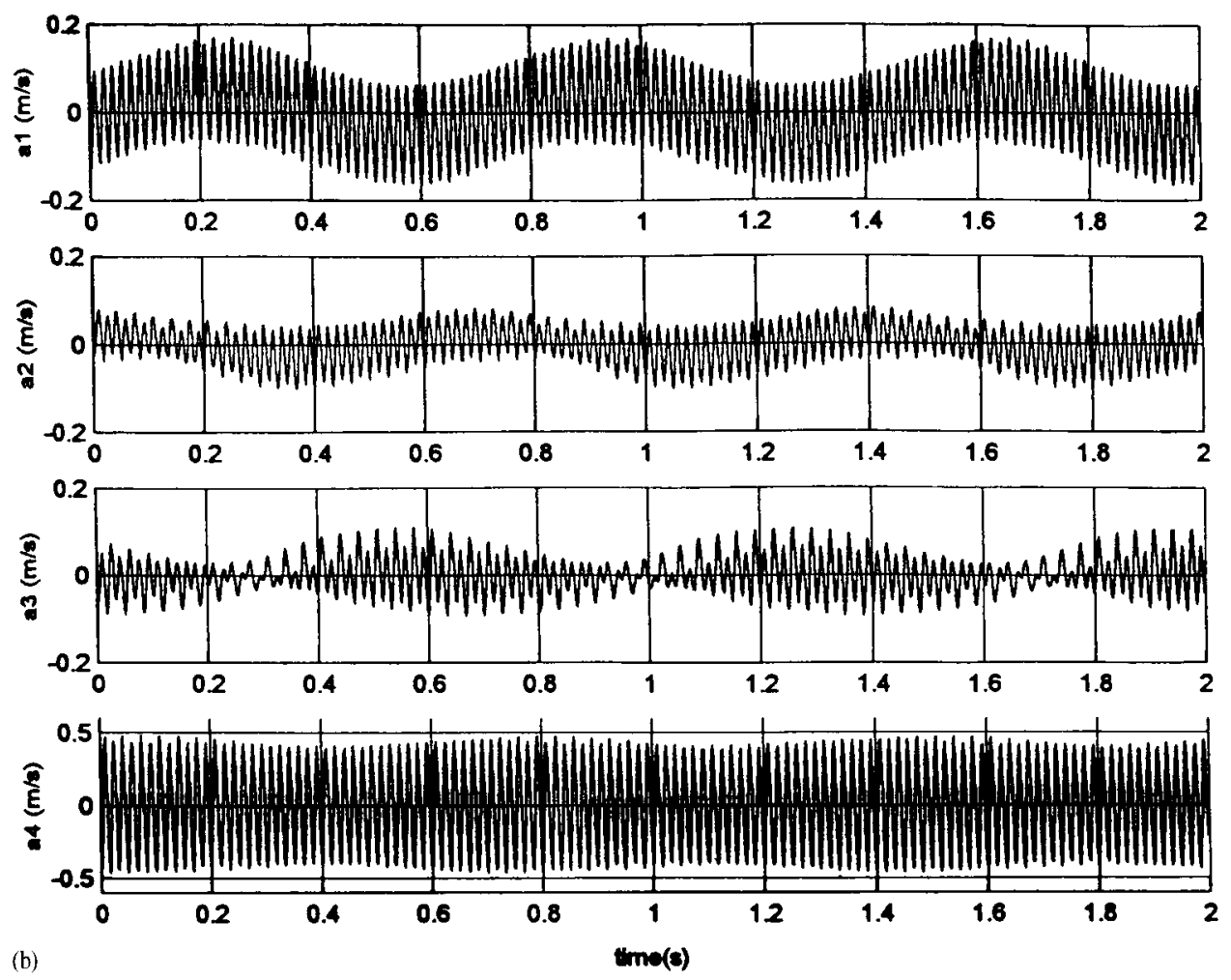

Fig. 17 (continued).

have a $180^{\circ}$ phase with respect to the fourth mode. Again Figs. 14(b) and 15(b) show that the concept of non-linear normal modes is questionable.

Figs. 16(a) and (b) show 18 ( 9 light lines and then 9 dark lines) consecutive curve-fitted velocity profiles of the beam excited at $60 \mathrm{~Hz}$, and the modal velocities $a_{i}$, respectively. Fig. 16(b) shows that the first mode motion consists of 60 and $1.406 \mathrm{~Hz}\left(=\omega_{1}\right)$ harmonics, and the second, third, and fourth modes vibrate at $60 \mathrm{~Hz}$ but their amplitudes modulate at $1.406 \mathrm{~Hz}$. Figs. 17(a) and (b) shows 18 (9 light lines and then 9 dark lines) consecutive curve-fitted velocity profiles of the beam excited at $60 \mathrm{~Hz}$ with a base excitation larger than that in Fig. 16, and the modal velocities $a_{i}$, respectively. The three sets of velocity profiles are separated by $0.5 \pi / \omega_{1}$, and they show the influence of the first mode. Fig. 17(b) shows that the first mode motion consists of 60 and $1.406 \mathrm{~Hz}$ harmonics, the second and fourth modes vibrate at $60 \mathrm{~Hz}$ but their amplitudes modulate at $1.406 \mathrm{~Hz}$, and the third mode motion consists of 60 and $30 \mathrm{~Hz}$ harmonics with amplitudes modulating at $1.406 \mathrm{~Hz}$.

When the beam is set downward, its dynamic responses are similar to those of the upward beam except that the resonance frequencies increase a little. Figs. 18(a) and (b) show 18 (9 light lines and then 9 dark lines) consecutive curve-fitted velocity profiles of the beam excited at $60 \mathrm{~Hz}$, and the modal velocities $a_{i}$, respectively. Fig. 18(b) shows that each of the four modes consists of 60 and $1.875 \mathrm{~Hz}\left(=\omega_{1}\right)$ harmonics. 

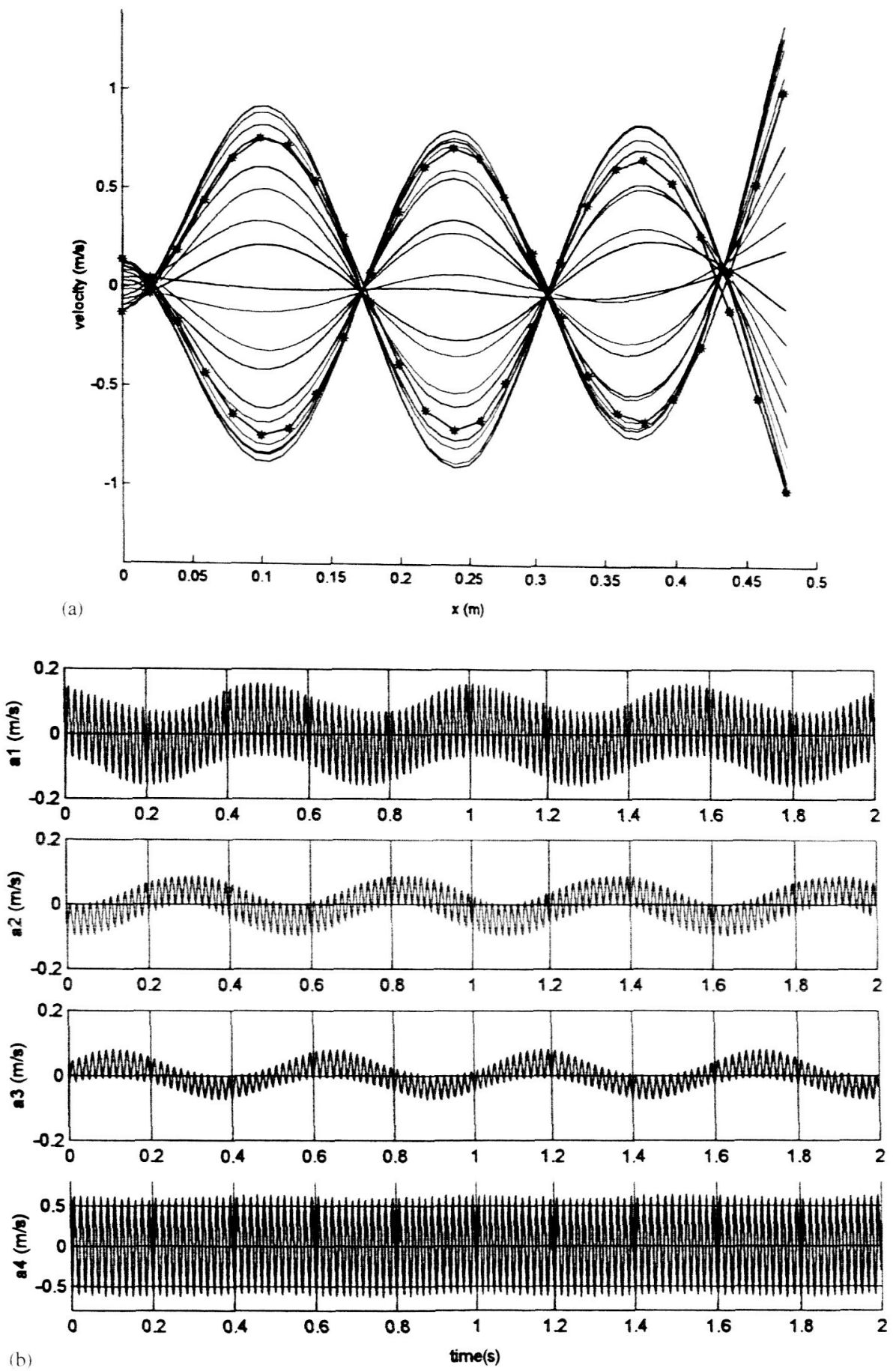

Fig. 18. 18 velocity profiles at consecutive time steps when the beam is set downward. $\Omega=60 \mathrm{~Hz}$. and $\dot{b}_{m u x}=0.1332 \mathrm{~m} / \mathrm{s}:$ (a) curve-fitted data and numerical prediction (stars), and (b) modal velocities. 
The existence of the first mode vibrating at $\omega_{1}$ in Figs. 12(d), 16(b), 17(b), and 18(b) is due to the so-called energy transfer from a directly excited high-frequency mode to a very low-frequency mode which is not directly or indirectly excited through internal or external resonances [11]. The existence of 1:2 internal resonance in Figs. 9(b), 10(b), 11(b), 12(c), and 17(b) shows that Eq. (8) cannot be used to predict the dynamics of this beam because Eq. (8) cannot account for quadratic non-linearities due to initial imperfection. The $k_{2}$ in Eq. (1) can be used to account for initial imperfection, and the $q_{3}$ can be used to account for part of the structural weight if the beam is not vertical.

\section{Concluding remarks}

We presented a method for the characterization of non-linear structural dynamics using structural velocities measured by a scanning laser vibrometer. Dynamics characterization of structures using a shaker and a scanning laser vibrometer is examined in detail, and several factors that may affect the characterization are discussed. Experimental results indicate that the use of non-linear normal modes in analyzing structural dynamics is questionable. Moreover, experimental results show the existence of $1: 3,1: 2: 3,1: 6$, and 1:2 external/internal resonances, amplitude-modulated motion, and energy transfer. This method is also valid for non-linear dynamics characterization of two-dimensional structures.

\section{Acknowledgements}

This work is supported by the National Science Foundation through Grants CMS-9871288 and CMS-9912482 and the NASA Langley Research Center through Grant NAG-1-01037. The support is gratefully acknowledged.

\section{References}

[1] M. Salama, M. Lou, H. Fang, Deployment of inflatable space structures: a review of recent developments, AIAA2000-1730, 2000.

[2] G. Greschik, M.M. Mikulas, A. Palisoc, Approximations and errors in pressurized axisymmetric membrane shape predictions, AIAA-98-2101, 1998.

[3] D. Gorinevsky, T. Hyde, C. Cabuz, Distributed localized shape control of Gossamer space structures, AIAA-2001$1197,2001$.

[4] Y. Goto, Y. Watanabe, T. Kasugai, M. Obata, Elastic buckling phenomenon applicable to deployable rings, International Journal of Solids and Structures 29 (1992) 893-909.

[5] P.F. Pai, A.N. Palazotto, Large-deformation analysis of flexible beams, International Journal of Solids and Structures 33 (1996) 1335-1353.

[6] P.F. Pai, T.J. Anderson, E.A. Wheater, Large-deformation tests and total-Lagrangian finite-element analyses of flexible beams, International Journal of Solids and Structures 37 (2000) 2951-2980.

[7] P.F. Pai, A.H. Nayfeh, A fully non-linear theory of curved and twisted composite rotor blades accounting for warpings and three-dimensional stress effects, International Journal of Solids and Structures 31 (1994) 1309-1340.

[8] J. Stoer, R. Bulirsch, Introduction to Numerical Analysis, Springer, New York, 1980. 
[9] P.F. Pai, A.H. Nayfeh, Nonlinear non-planar oscillations of a cantilever beam under lateral base excitations, International Journal of Nonlinear Mechanics 25 (5) (1990) 455-474.

[10] A.H. Nayfeh. Perturbation Methods. Wiley-Interscience. New York, 1973.

[11] A.H. Nayfeh. S.A. Nayfeh, T.A. Anderson. B. Balachandran. Transfer of energy from high-frequency to lowfrequency modes, in: Nonlinearity and Chaos in Engineering Dynamics, Wiley-Interscience, New York, 1994, pp. $39-58$. 